\title{
Keldysh field theory for nonequilibrium condensation in a parametrically pumped polariton system
}

\author{
K. Dunnett and M. H. Szymańska* \\ Department of Physics and Astronomy, University College London, Gower Street, London, WC1E 6BT, United Kingdom
}

(Received 4 January 2016; revised manuscript received 17 March 2016; published 10 May 2016)

\begin{abstract}
We develop a quantum field theory for parametrically pumped polaritons using Keldysh Green's function techniques with which the occupations of the excitation spectra can be calculated. By considering the mean field and Gaussian fluctuations, we find that the highly nonequilibrium phase transition to the optical parametric oscillator regime is in some ways similar to equilibrium condensation. In particular, we show that this phase transition can be associated with an effective chemical potential, at which the system's bosonic distribution function diverges, and an effective temperature for low energy modes. As in equilibrium systems, the transition is achieved by tuning this effective chemical potential to the energy of the lowest normal mode. Since the nonequilibrium occupations of the modes are available, we determine experimentally observable properties such as the luminescence and absorption spectra.
\end{abstract}

DOI: 10.1103/PhysRevB.93.195306

\section{INTRODUCTION}

Phase transitions in driven-dissipative systems of light strongly coupled to matter have been the subject of much recent study and progress [1]. Their intrinsic nonequilibrium nature, caused by the decay of photons, results in phase transitions that may differ from their equilibrium analogues $[1,2]$. In many cases, a high degree of control of the system is possible, and questions relating to nonequilibrium statistical mechanics and the interplay between equilibrium and nonequilibrium behaviours can be addressed [3-8]. Polaritons confined to two dimensions in semiconductor microcavities are a well studied example, showing quantum condensation $[9,10]$, superfluidity [11-14], and rich hydrodynamics [11,15-20].

Several studies of polaritons have considered the case of incoherent pumping, where carriers are injected at high energies or momenta and undergo a complex process of exciton formation and subsequent scattering to relax to the ground state at the bottom of the lower polariton dispersion [1]. This scheme was particularly appealing in the search for spontaneous equilibrium Bose-Einstein condensation (BEC) of polaritons $[9,10]$. However, it leads to a large dynamical exciton reservoir which affects polariton condensation [21] and superfluidity. The complicated pumping and relaxation processes are not well understood, and models based on phenomenological descriptions suffer from inconsistencies and divergences [22,23].

Polaritons can also be excited directly by coherent pumping with a monochromatic laser tuned to the lower polariton dispersion [24]. Under certain pumping conditions, a coherently pumped polariton system undergoes a phase transition to an optical parametric oscillator (OPO) regime in which pairs of pump polaritons parametrically scatter into new signal and idler quasicondensate states [25-27]. Without the exciton reservoir, the theoretical treatment is simplified and an (almost) $a b$ initio description is possible [28-35]. In the coherently pumped configuration there are two distinct regimes: (i) the regime where only the polariton mode close in energy and momentum to the external pump is largely occupied,

*m.szymanska@ucl.ac.uk which is the "coherently pumped" regime; (ii) the OPO or "parametrically pumped" regime where three modes (the signal, pump, and idler), distinct in energy and momentum, are largely occupied. These definitions are used throughout this paper.

Coherently pumped polariton systems have been used to explore polariton superfluidity by examining scattering against a defect [12] since flows with any wave vector can be initiated easily. However, under coherent pumping, the phase of the polariton field is locked to the applied pump, which explicitly breaks the $U(1)$ phase symmetry and results in a gapped excitation spectrum [33]. Since this is fundamentally different to what is expected for a superfluid [36], the interpretation of the reduced drag when passing an obstacle at small velocity as strong evidence for a superfluid becomes questionable.

Meanwhile, in the OPO regime, the relative phase of the signal and idler states is free and the $U(1)$ phase symmetry is spontaneously broken in each realisation. The excitation spectrum now possesses a gapless Goldstone mode [35] exactly as in the incoherently pumped case. Such a system is expected to be a superfluid according to the traditional definitions. A recent theoretical and experimental study of flows past an obstacle in polariton OPO has shown that the coupling between the three OPO modes leads to rich nonlinear behavior [14].

To date, the parametrically pumped polariton system has been described in terms of dynamical equations [28-35], which reflects that polariton OPO is a purely nonequilibrium phenomenon; in the absence of drive and decay, no phase transition would exist. The mean field steady states and the excitation spectra both above and below the OPO threshold have been studied [32-35], and quantum Langevin equations have been used to calculate the photoluminescence below threshold [28,30]. A recent analysis using truncated Wigner methods has shown that the OPO transition is of the Berezinskii-Kosterlitz-Thouless (BKT) type and is associated with the binding and proliferation of vortex-anti-vortex pairs [37]. The truncated Wigner methods, however, do not give easy access to correlations at different times and so energy resolved properties, such as the luminescence and absorption spectra, are difficult to obtain. 
In this paper, we develop a quantum field theory for coherently pumped polaritons across the OPO threshold using Keldysh Green's function techniques, which allows the calculation of all two time correlation functions including the occupations of all modes [38-40]. The Keldysh functional integral approach has been used to study the phase transitions of several driven-dissipative systems including the superradiant and glassy phase transitions of the Dicke model [8,41], BEC of photons in dye-filled cavities [42], atoms in multimode cavities [43], a 1-D driven dissipative system near quantum criticality [44], and exciton polaritons in semiconductor microcavities under incoherent excitation [3$6,45,46]$. It also allows direct comparison with equilibrium phase transitions [39].

We find that despite the highly nonequilibrium nature of the coherently pumped polariton system, effective thermal quantities can be defined below the OPO phase transition, and that the approach to the transition is similar to that in an equilibrium system. In particular, we find that that the system's distribution function diverges at a specific energy, leading to an effective chemical potential [4,5]. Moreover, the divergence has the form 1/energy, which leads to the definition of an effective temperature [41]. As in equilibrium, the phase transition is achieved by tuning the effective chemical potential to the energy of the lowest normal mode [5].

This paper is organized as follows: The polariton system and model are introduced in Sec. II, and in Sec. III the Keldysh formalism for the model is set up without making any approximations about the number of modes. In Sec. IV, we consider the coherently pumped system in the pump only, or "normal," state and discuss the approach to the transition discussed, including the concepts of an effective temperature and chemical potential. The OPO regime, with the "quasicondensed" signal and idler modes, is considered in Sec. V, and conclusions are in Sec. VI.

\section{POLARITON SYSTEM AND MODEL}

In this section the driven-dissipative polariton system is described, and we introduce a general Hamiltonian.

\section{A. Polaritons}

Microcavity polaritons are the quasiparticles resulting from strong coupling between quantum well excitons (bound electron-hole pairs) and confined photons in semiconductor microcavities when the exciton-photon interactions are greater than the losses [1,47]. Diagonalizing the Hamiltonian describing a lossless exciton-photon system leads to the new bosonic eigenstates [48], the upper and lower polaritons with dispersions $[1,2,49] \omega_{u p, l p}(\mathbf{k})=$ $\frac{1}{2}\left(\omega_{x}(\mathbf{k})+\omega_{c}(\mathbf{k}) \pm \sqrt{\left(\omega_{x}(\mathbf{k})-\omega_{c}(\mathbf{k})\right)^{2}+\Omega_{R}^{2}}\right)$, where $\omega_{x}(\mathbf{k})$ and $\omega_{c}(\mathbf{k})$ are the exciton and photon dispersions, respectively, and $\Omega_{R}$ is the Rabi frequency describing the strength of the coupling. The polariton operators (upper: $\hat{b}_{\mathbf{k}}$; lower: $\hat{a}_{\mathbf{k}}$ ) can be written in terms of the exciton $\hat{x}_{\mathbf{k}}$ and photon $\hat{c}_{\mathbf{k}}$ operators

$$
\begin{aligned}
& \hat{a}_{\mathbf{k}}=X(\mathbf{k}) \hat{x}_{\mathbf{k}}+C(\mathbf{k}) \hat{c}_{\mathbf{k}}, \\
& \hat{b}_{\mathbf{k}}=-C(\mathbf{k}) \hat{x}_{\mathbf{k}}+X(\mathbf{k}) \hat{c}_{\mathbf{k}},
\end{aligned}
$$

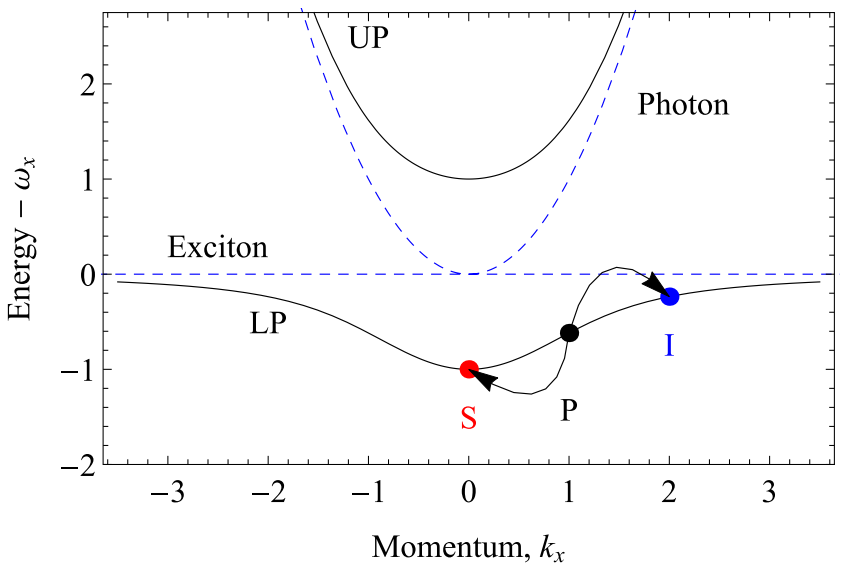

FIG. 1. Dispersions: UP (upper polariton), LP (lower polariton), exciton and cavity photon. All units are nondimensional. Pairs of lower polaritons introduced by an external pump, P (black), scatter coherently into the signal, $\mathrm{S}$ (red), and idler, I (blue) states while conserving energy and momentum. OPO with $k_{s} \approx 0$ is observed for a range of pump energies and momenta [51].

where $X(\mathbf{k})$ and $C(\mathbf{k})$ are the excitonic and photonic Hopfield coefficients [30,49]:

$$
\begin{aligned}
X(\mathbf{k}) & =\frac{\omega_{l p}(\mathbf{k})-\omega_{c}(\mathbf{k})}{\sqrt{\left(\omega_{l p}(\mathbf{k})-\omega_{c}(\mathbf{k})\right)^{2}+\left(\frac{\Omega_{R}}{2}\right)^{2}}}, \\
C(\mathbf{k}) & =\frac{\Omega_{R}}{2 \sqrt{\left(\omega_{l p}(\mathbf{k})-\omega_{c}(\mathbf{k})\right)^{2}+\left(\frac{\Omega_{R}}{2}\right)^{2}}},
\end{aligned}
$$

which are normalized to $X^{2}(\mathbf{k})+C^{2}(\mathbf{k})=1 . X^{2}(\mathbf{k})$ and $C^{2}(\mathbf{k})$ give the exciton and photon fractions of the lower polaritons, respectively.

The cavity photons are much lighter than the excitons $\left(m_{c} \approx 2.3 \times 10^{-5} m_{e}, m_{x} \approx 0.3 m_{e}\right.$, with $m_{e}$ the free electron mass), so the exciton dispersion is usually assumed flat while the cavity photon dispersion is parabolic $\omega_{c}(\mathbf{k})=\omega_{c}(0)+$ $|\mathbf{k}|^{2} / 2 m_{c}$. The minimum separation of the two polariton branches is $\Omega_{R}$, which occurs at $\mathbf{k}=0$ for $\omega_{c}(0)=\omega_{x}$, as shown in Fig. 1 [2,49,50]. Different exciton-photon detunings are accessible by choice of position on the wedge shaped samples used in experiments [49].

As mentioned in the introduction, polaritons are created by applying a coherent laser pump near the point of inflection of the lower polariton dispersion. Above a threshold pump strength, pairs of pump polaritons spontaneously scatter into new 'signal' $\left(\mathbf{k}_{s}<\mathbf{k}_{p}, \omega_{s}<\omega_{p}\right)$ and 'idler' $\left(\mathbf{k}_{i}>\mathbf{k}_{p}, \omega_{i}>\right.$ $\left.\omega_{p}\right)$ states that become extensively occupied. Energy and momentum are conserved in the scattering process: $2 \omega_{p}=$ $\omega_{s}+\omega_{i}, 2 \mathbf{k}_{p}=\mathbf{k}_{s}+\mathbf{k}_{i}$. This is only possible due to the excitonic nonlinearity of the lower polaritons [30] and is called the optical parametric oscillator (OPO) regime in analogy to nonlinear optics [26]. The OPO scattering is shown schematically in Fig. 1. For a given set of pump parameters, the observed signal and idler modes are at well defined energies and momenta [51] and have a large degree of temporal and spatial coherence [26]. 


\section{B. Hamiltonian}

We start from a Hamiltonian

$$
\hat{H}_{l p}=\hat{H}_{0}+\hat{H}_{\text {int }}+\hat{H}_{\text {pump }}+\hat{H}_{\text {decay }},
$$

describing interacting lower polaritons $\left(\hat{a}_{\mathbf{k}}, \hat{a}_{\mathbf{k}}^{\dagger}\right)$

$$
\begin{aligned}
\hat{H}_{0} & =\sum_{\mathbf{k}} \omega_{l p}(\mathbf{k}) \hat{a}_{\mathbf{k}}^{\dagger} \hat{a}_{\mathbf{k}}, \\
\hat{H}_{\mathrm{int}} & =\sum_{\mathbf{k}, \mathbf{k}^{\prime}, \mathbf{q}} \frac{V_{\mathbf{k}, \mathbf{k}^{\prime}, \mathbf{q}}}{2} \hat{a}_{\mathbf{k}}^{\dagger} \hat{a}_{\mathbf{k}^{\prime}}^{\dagger} \hat{a}_{\mathbf{k}-\mathbf{q}} \hat{a}_{\mathbf{k}^{\prime}+\mathbf{q}},
\end{aligned}
$$

driven by a coherent pump $\left(F_{p}\right)$ of the form

$$
F_{p}=f e^{-i \omega_{p} t} e^{i \mathbf{k}_{p} \cdot \mathbf{x}},
$$

which introduces polaritons at a single energy $\omega_{p}$ and momentum $\mathbf{k}_{p}$,

$$
\hat{H}_{\text {pump }}=\hat{a}_{\mathbf{k}_{p}}^{\dagger} F_{p}+F_{p}^{\star} \hat{a}_{\mathbf{k}_{p}},
$$

and coupled with strength $\Gamma_{\mathbf{p}}^{\mathbf{k}}$ to a bosonic decay bath $\hat{A}_{\mathbf{p}}$ with dispersion $\omega_{\mathbf{p}}^{\Gamma}=\omega^{\Gamma}(\mathbf{p})$

$$
\hat{H}_{\text {decay }}=\sum_{\mathbf{k}, \mathbf{p}} \Gamma_{\mathbf{p}}^{\mathbf{k}}\left(\hat{a}_{\mathbf{k}}^{\dagger} \hat{A}_{\mathbf{p}}+\hat{A}_{\mathbf{p}}^{\dagger} \hat{a}_{\mathbf{k}}\right)+\sum_{\mathbf{p}} \omega_{\mathbf{p}}^{\Gamma} \hat{A}_{\mathbf{p}}^{\dagger} \hat{A}_{\mathbf{p}} .
$$

Each polariton mode with $2 \mathrm{D}$ momentum $\mathbf{k}$ couples to an independent set of decay bath modes with momenta $\mathbf{p}$, conserving in plane momentum [4]. The restriction to lower polaritons is valid so long as only moderate values of pumping are used and the pump is always applied close to the lower polariton curve $\left(\omega_{p} \approx \omega_{l p}\left(\mathbf{k}_{p}\right)\right)$ [35], ensuring that nonlinear terms involving both polariton branches are negligible [32].

The polariton-polariton interaction strength is given by:

$$
V_{\mathbf{k}, \mathbf{k}^{\prime}, \mathbf{q}}=g_{\mathrm{x}} X(\mathbf{k}) X\left(\mathbf{k}^{\prime}\right) X(\mathbf{k}-\mathbf{q}) X\left(\mathbf{k}^{\prime}+\mathbf{q}\right),
$$

where the $X(\mathbf{k})$ 's are defined in Eq. (1) and reflect that the polariton interactions are due to the excitonic component of the polaritons. While the exciton-exciton interaction strength $g_{\mathrm{x}}$ can be assumed constant, the polariton-polariton interaction is in general momentum dependent. For $\omega_{c}(0)=\omega_{x}, 1 / 2 \leqslant$ $X^{2}(\mathbf{k}) \leqslant 1[49]$.

To proceed further it is helpful to first perform a gauge transformation, which effectively moves us to the reference frame of the pump mode and makes the Hamiltonian time independent. We define the new operator $\tilde{a}=\hat{a} e^{i \omega_{p} t} e^{-i \mathbf{k}_{p} \cdot \mathbf{x}}$ and similarly $\tilde{a}^{\dagger}$. Without loss of generality, $f \in \mathfrak{R}$ in Eq. (4) can be chosen so the pump term becomes:

$$
\hat{H}_{\text {pump }} \rightarrow f\left(\tilde{a}_{\mathbf{0}}+\tilde{a}_{\mathbf{0}}^{\dagger}\right) .
$$

To write the entire Hamiltonian with the new operators $(\tilde{a})$, we note that $\hat{a}=\tilde{a} e^{-i \omega_{p} t} e^{i \mathbf{k}_{p} \cdot \mathbf{x}}$ and that the exponents cancel in all terms of the Hamiltonian that have the form $\hat{a}^{\dagger} \hat{a}$ and $\hat{a}^{\dagger} \hat{a}^{\dagger} \hat{a} \hat{a}$. What is left is the momentum $\mathbf{k}_{p}$ shift coming from the kinetic energy term.

The terms that contain the decay bath are now:

$$
\begin{aligned}
\hat{H}_{\text {decay }}= & \sum_{\mathbf{p}} \omega_{\mathbf{p}}^{\Gamma} \hat{A}_{\mathbf{p}}^{\dagger} \hat{A}_{\mathbf{p}}+\sum_{\mathbf{k}, \mathbf{p}} \Gamma_{\mathbf{p}}^{\mathbf{k}}\left(\tilde{a}_{\mathbf{k}}^{\dagger} e^{i \omega_{p} t} e^{-i \mathbf{k}_{p} \cdot \mathbf{x}} \hat{A}_{\mathbf{p}}\right. \\
& \left.+\hat{A}_{\mathbf{p}}^{\dagger} \tilde{a}_{\mathbf{k}} e^{-i \omega_{p} t} e^{i \mathbf{k}_{p} \cdot \mathbf{x}}\right) .
\end{aligned}
$$

By defining $\hat{A}=\tilde{A} e^{-i \omega_{p} t} e^{i \mathbf{k}_{p} \cdot \mathbf{x}}$ (exactly as the polariton operator), the entire system is written relative to the pump energy $\omega_{p}$ and momentum $\mathbf{k}_{p}$, and the explicit time dependence of Eq. (5) is removed.

\section{KELDYSH FORMALISM}

The Keldysh functional integral approach is now applied to both coherently and parametrically pumped polaritons. In this section, we obtain a general complex Gross-Pitaevskii equation (cGPE) describing the mean field for any ansatz without restricting the form of the solution. The process of including fluctuations is summarized, and all quantities of interest are defined.

\section{A. The functional integral representation}

The basis of the functional integral approach is the partition function of the system that can be written as a coherent state path integral over bosonic fields $\bar{\psi}, \psi[38-40]$ :

$$
Z=N \int D(\bar{\psi}, \psi) e^{i S},
$$

where $N$ provides the correct normalization and $S$ is the action: $S=S[\bar{\psi}, \psi]$. In the Keldysh formalism, the system is considered to evolve from the distant past $(t=-\infty)$ to the distant future $(t=+\infty)$ on the forwards branch of a closed time contour and then return along the backwards branch. The time evolution on the two branches is written in terms of the separate fields on each branch, $\psi_{f, b}$, and then rotated into a quantum-classical basis [38-40]:

$$
\psi_{f, b}=\frac{1}{\sqrt{2}}\left(\psi_{c l} \pm \psi_{q}\right) .
$$

We write the action as a sum over elements corresponding to the parts of the Hamiltonian, Eq. (3):

$$
i S=i \int d t\left(S_{0}+S_{\mathrm{int}}+S_{\mathrm{pump}}+S_{\mathrm{decay}}\right) .
$$

The components are:

$$
\begin{aligned}
S_{0}= & \sum_{\mathbf{k}} \Psi_{\mathbf{k}}^{\dagger}(t)\left(i \partial_{t}-\omega_{l p}\left(\mathbf{k}+\mathbf{k}_{\mathbf{p}}\right)+\omega_{p}\right) \hat{\sigma}_{1}^{K} \Psi_{\mathbf{k}}(t) \\
S_{\mathrm{int}}= & -\sum_{\mathbf{k}, \mathbf{k}^{\prime}, \mathbf{q}} \frac{V_{\mathbf{k}, \mathbf{k}^{\prime}, \mathbf{q}}}{4}\left(\Psi_{\mathbf{k}}^{\dagger}(t) \hat{\sigma}_{1}^{K} \Psi_{\mathbf{k}-\mathbf{q}}(t) \Psi_{\mathbf{k}^{\prime}}^{\dagger}(t) \Psi_{\mathbf{k}^{\prime}+\mathbf{q}}(t)\right. \\
& \left.+\Psi_{\mathbf{k}}^{\dagger}(t) \Psi_{\mathbf{k}-\mathbf{q}}(t) \Psi_{\mathbf{k}^{\prime}}^{\dagger}(t) \hat{\sigma}_{1}^{K} \Psi_{\mathbf{k}^{\prime}+\mathbf{q}}(t)\right) \\
& S_{\text {pump }}=-\sqrt{2} f\left(\bar{\psi}_{\mathbf{0}, q}(t)+\psi_{\mathbf{0}, q}(t)\right) \\
S_{\text {decay }}= & \sum_{\mathbf{p}} \chi_{\mathbf{p}}^{\dagger}(t)\left(i \partial_{t}-\omega_{\mathbf{p}+\mathbf{k}_{p}}^{\Gamma}+\omega_{p}\right) \hat{\sigma}_{1}^{K} \chi_{\mathbf{p}}(t) \\
& -\sum_{\mathbf{k}, \mathbf{p}} \Gamma_{\mathbf{p}}^{\mathbf{k}}\left(\chi_{\mathbf{p}}^{\dagger}(t) \hat{\sigma}_{1}^{K} \Psi_{\mathbf{k}}(t)+\Psi_{\mathbf{k}}^{\dagger}(t) \hat{\sigma}_{1}^{K} \chi_{\mathbf{p}}(t)\right)
\end{aligned}
$$

where $\hat{\sigma}_{1}^{K}$ is the Pauli matrix [52] in the Keldysh quantumclassical space, and the bath $\chi$ and polariton $\Psi$ fields are 
written as vectors of the quantum and classical fields:

$\hat{\sigma}_{1}^{K}=\left(\begin{array}{ll}0 & 1 \\ 1 & 0\end{array}\right), \quad \Psi_{\mathbf{k}}(t)=\left(\begin{array}{c}\psi_{\mathbf{k}, c l}(t) \\ \psi_{\mathbf{k}, q}(t)\end{array}\right), \quad \chi_{\mathbf{p}}(t)=\left(\begin{array}{c}\chi_{\mathbf{p}, c l}(t) \\ \chi_{\mathbf{p}, q}(t)\end{array}\right)$.

Since the pump is classical, i.e., the same on the forward and backward branches of the Keldysh contour, after the Keldysh rotation it only couples to quantum fields in Eq. (11). Moreover, the pump injects polaritons with momentum $\mathbf{k}_{p}$ which, after the gauge transformation to the pump frame, corresponds to zero momentum in Eq. (11). The momentum $\mathbf{k}_{p}$ and energy $\omega_{p}$ shifts in Eqs. (9) and (12) come from the spatial (Fourier transform of the polariton and the bath's dispersion operators) and time derivatives acting on $\hat{a}_{\mathbf{k}}=\tilde{a}_{\mathbf{k}} e^{-i \omega_{p} t} e^{i \mathbf{k}_{p} \cdot \mathbf{x}}$ and $\hat{A}=\tilde{A} e^{-i \omega_{p} t} e^{i \mathbf{k}_{p} \cdot \mathbf{x}}$, respectively.

\section{B. Integrating out the decay bath}

The bath fields are present in the action at quadratic level so the functional integral over them can be performed analytically $[4,43]$. The procedure is the same as for the photon decay bath in earlier studies of driven-dissipative polaritons [4]. After performing the Gaussian integration over the decay bath's fields, we Fourier transform into the energy representation and make a series of standard assumptions about the properties of the decay bath [4].

In particular, we assume that the bath couples equally to all polariton modes $\hat{a}_{\mathbf{k}}, \Gamma_{\mathbf{p}}^{\mathbf{k}} \rightarrow \Gamma_{\mathbf{p}}$, so the polariton decay is momentum independent. To include full momentum dependence in the polariton decay, one would either have to explicitly include momentum dependent coupling here, presupposing knowledge of the correct Hopfield coefficient weightings, or consider two independent baths of excitons and photons which would be weighted properly as a result of the rotation from the exciton-photon basis.

Further, the decay bath is assumed to be large and unaffected by the behavior of the system, that the coupling $\Gamma_{\mathbf{p}}$ is a smooth function of $\mathbf{p}$, and that the bath has a dense energy spectrum, so the summation over $\mathbf{p}$ can be replaced by $\int d \omega^{\Gamma}$. Although we could choose any form of the decay bath's density of states and coupling to the polariton system, it is reasonable to assume that they are constant. This leads to self-energy contributions from the decay bath [4]:

$$
\begin{aligned}
d^{R, A}(\omega) & =\mp i \kappa_{l p}, \\
d^{K}(\omega) & =-2 i \kappa_{l p} F_{\chi}\left(\omega+\omega_{p}\right),
\end{aligned}
$$

where $\kappa_{l p}$ is the constant polariton decay rate and $F_{\chi}(\omega)$ is the bath's distribution function. The presence of $\omega_{p}$ is due to gauge transformation to the pump frame. Note that since there is still an explicit $\omega$ dependence in the bath distribution present in the Keldysh part of the self-energy, the influence of the bath is non-Markovian at this stage. However, as discussed in detail in Ref. [41], since the drive frequency $\omega_{p}$ is much larger then the energy associated with the room temperature thermal photons outside the cavity, decay bath modes with energies in the range of interest $|\omega|<\Omega_{R}$ are effectively not occupied, and $F_{\chi}\left(\omega+\omega_{p}\right)$ can be set to 1 . However, if needed, inclusion of a frequency dependent decay bath in both the retarded (deterministic in the case of Wigner approach [53]) and Keldysh (stochastic) components is straightforward in the Keldysh formalism.

The decay term in the Keldysh action now contains only the polariton fields, and after inverting the Fourier transform, Eq. (12) is replaced by:

$$
\begin{aligned}
S_{\text {decay }}= & \kappa_{l p} \sum_{\mathbf{k}} \int d t \Psi_{\mathbf{k}}^{\dagger}(t) \hat{\sigma}_{2}^{K} \Psi_{\mathbf{k}}(t) \\
& +2 i \kappa_{l p} \sum_{\mathbf{k}} \int d t d t^{\prime} \bar{\psi}_{\mathbf{k}, q}(t) F_{\chi}\left(t-t^{\prime}\right) \psi_{\mathbf{k}, q}\left(t^{\prime}\right),
\end{aligned}
$$

where $\hat{\sigma}_{2}^{K}$ is the Pauli matrix

$$
\hat{\sigma}_{2}^{K}=\left(\begin{array}{cc}
0 & -i \\
i & 0
\end{array}\right)
$$

\section{Mean field and saddle points}

The mean field equation is calculated from the saddle points of the action $[4,38,39]$ taken relative to both the classical and quantum fields. This involves finding the solutions to

$$
\frac{\partial S}{\partial \bar{\psi}_{\mathbf{k}, c l}(t)}=0 \quad \text { and } \quad \frac{\partial S}{\partial \bar{\psi}_{\mathbf{k}, q}(t)}=0,
$$

which leads to

$$
\begin{aligned}
\frac{\partial S}{\partial \bar{\psi}_{\mathbf{k}, c l}(t)}= & \int d t\left[\left(i \partial_{t}-\omega_{l p}\left(\mathbf{k}+\mathbf{k}_{p}\right)+\omega_{p}-i \kappa_{l p}\right) \psi_{\mathbf{k}, q}(t)\right. \\
& -\sum_{\mathbf{k}^{\prime}, \mathbf{q}} \frac{V_{\mathbf{k}, \mathbf{k}^{\prime}, \mathbf{q}}}{2}\left(\bar{\psi}_{\mathbf{k}^{\prime}, q}(t) \Psi_{\mathbf{k}-\mathbf{q}}^{T}(t) \Psi_{\mathbf{k}^{\prime}+\mathbf{q}}(t)\right. \\
& \left.\left.+\bar{\psi}_{\mathbf{k}^{\prime}, c l}(t) \Psi_{\mathbf{k}-\mathbf{q}}^{T}(t) \hat{\sigma}_{1}^{K} \Psi_{\mathbf{k}^{\prime}+\mathbf{q}}(t)\right)\right]
\end{aligned}
$$

and

$$
\begin{aligned}
\frac{\partial S}{\partial \bar{\psi}_{\mathbf{k}, q}(t)}= & \int d t\left[\left(i \partial_{t}-\omega_{l p}\left(\mathbf{k}+\mathbf{k}_{p}\right)+\omega_{p}+i \kappa_{l p}\right) \psi_{\mathbf{k}, c l}(t)\right. \\
& -\sum_{\mathbf{k}^{\prime}, \mathbf{q}} \frac{V_{\mathbf{k}, \mathbf{k}^{\prime}, \mathbf{q}}}{2}\left(\bar{\psi}_{\mathbf{k}^{\prime}, q}(t) \Psi_{\mathbf{k}-\mathbf{q}}^{T}(t) \hat{\sigma}_{1}^{K} \Psi_{\mathbf{k}^{\prime}+\mathbf{q}}(t)\right. \\
& \left.\left.+\bar{\psi}_{\mathbf{k}^{\prime}, c l}(t) \Psi_{\mathbf{k}-\mathbf{q}}^{T}(t) \Psi_{\mathbf{k}^{\prime}+\mathbf{q}}(t)\right)-\sqrt{2} f \delta_{\mathbf{k}, 0}\right] \\
& -2 i \kappa_{l p} \iint d t d t^{\prime} F_{\chi}\left(t-t^{\prime}\right) \psi_{\mathbf{k}, q}\left(t^{\prime}\right) .
\end{aligned}
$$

There always exists a solution to the saddle point equations where the quantum part is zero, which corresponds to the purely classical solution and leads to the complex Gross-Pitaevskii equation (cGPE) of the mean field analysis $[4,38,39]$. With $\psi_{\mathbf{k}, \mathbf{q}}(t)=0, \partial S / \partial \bar{\psi}_{\mathbf{k}, \mathbf{c l}}(t)=0$ is automatically satisfied, and the integrand of the second equation gives

$$
\begin{aligned}
0= & \left(i \partial_{t}-\omega_{l p}\left(\mathbf{k}+\mathbf{k}_{p}\right)+\omega_{p}+i \kappa_{l p}\right) \psi_{\mathbf{k}, c l}(t) \\
& -\sqrt{2} f \delta_{\mathbf{k}, 0}-\sum_{\mathbf{k}^{\prime}, \mathbf{q}} \frac{V_{\mathbf{k}, \mathbf{k}^{\prime}, \mathbf{q}}}{2} \bar{\psi}_{\mathbf{k}^{\prime}, c l}(t) \psi_{\mathbf{k}-\mathbf{q}, c l}(t) \psi_{\mathbf{k}^{\prime}+\mathbf{q}, c l}(t) .
\end{aligned}
$$

$\psi_{c l}$ at the saddle point, $\psi_{c l}^{s p}$, is $\sqrt{2}$ times the mean field value $\psi^{\mathrm{mf}}$ as defined in other approximations [32,34]. The mean field polariton field is equal to the $\psi_{f}$, as defined by Eq. (7), 
at the saddle point $[4,38]$, and:

$$
\begin{aligned}
0= & \left(i \partial_{t}-\omega_{l p}\left(\mathbf{k}+\mathbf{k}_{p}\right)+\omega_{p}+i \kappa_{l p}\right) \psi_{\mathbf{k}}^{\mathrm{mf}}-f \delta_{\mathbf{k}, 0} \\
& -\sum_{\mathbf{k}^{\prime}, \mathbf{q}} V_{\mathbf{k}, \mathbf{k}^{\prime}, \mathbf{q}} \bar{\psi}_{\mathbf{k}^{\prime}}^{\mathrm{mf}} \psi_{\mathbf{k}-\mathbf{q}}^{\mathrm{mf}} \psi_{\mathbf{k}^{\prime}+\mathbf{q}}^{\mathrm{mf}} .
\end{aligned}
$$

As will be seen, when we restrict to the appropriate modes, the usual cGPEs describing the polariton OPO [32,34] are reproduced. In this paper we consider steady state solutions to Eq. (14) in the coherently pumped and OPO regimes.

\section{Fluctuations about the mean field}

Having found the mean field cGPEs and their steady state solutions, the next task is to consider whether the solutions of these equations are stable to small fluctuations close in energy and momentum to the mean field state $[32,35]$. A physical solution requires stability of the saddle point equations, so only if the state is stable can other quantities such as the luminescence be calculated [4,54]. Small fluctuations $\delta \psi_{c l}$ and $\delta \psi_{q}$ are added to the mean field, and we construct the inverse Green's functions by substituting

$$
\psi_{c l}=\psi_{c l}^{s p}+\delta \psi_{c l}=\sqrt{2} \psi^{\mathrm{mf}}+\delta \psi_{c l}
$$

and

$$
\psi_{q}=\delta \psi_{q}
$$

into Eqs. (9)-(11) and (13), and restricting to terms that are second order in the fluctuations. Since all quantities of interest will be in energy-momentum space, we perform the Fourier transform at the level of the action of fluctuations. The remaining part of the action then has the form:

$$
S[\Delta \Psi]=\int d \omega \sum_{\mathbf{k}} \Delta \Psi_{\mathbf{k}}^{\dagger}(\omega)\left(\begin{array}{cc}
0 & {\left[D^{-1}\right]^{A}} \\
{\left[D^{-1}\right]^{R}} & {\left[D^{-1}\right]^{K}}
\end{array}\right) \Delta \Psi_{\mathbf{k}}(\omega),
$$

where $\left[D^{-1}\right]^{\{R / A / K\}}=\left[D^{-1}\right]^{\{R / A / K\}}(\omega, \mathbf{k})$ and $R, A, K$ indicate the retarded, advanced, and Keldysh components of the inverse Green's function, respectively. The fluctuations are written using a Nambu vector form [4]:

$$
\Delta \Psi_{\mathbf{k}}(\omega)=\left(\begin{array}{c}
\delta \psi_{\mathbf{k}, c l}(\omega) \\
\delta \bar{\psi}_{-\mathbf{k}, c l}(-\omega) \\
\delta \psi_{\mathbf{k}, q}(\omega) \\
\delta \bar{\psi}_{-\mathbf{k}, q}(-\omega)
\end{array}\right),
$$

where the fluctuations are relative to the pump mode. If there is more than one mode, each $\delta \psi$ has the structure $\left(\delta \psi_{m 1}, \delta \psi_{m 2} \ldots\right)^{T}$ and the fluctuations are relative to each mode $\left(_{ \pm \mathbf{k}}( \pm \omega) \rightarrow_{\mathbf{k}_{m} \pm \mathbf{k}}\left(\omega_{m} \pm \omega\right)\right)$. Further, the following relations are used $[38,40]$ :

$$
\begin{gathered}
{\left[D^{-1}\right]^{R}=\left[D^{R}\right]^{-1},} \\
D^{A}=\left(D^{R}\right)^{\dagger}, \\
D^{K}=-D^{R}\left[D^{-1}\right]^{K} D^{A}=D^{R} F_{s}-F_{s} D^{A},
\end{gathered}
$$

where $F_{s}$, Fourier transformed into energy, is the distribution matrix of the system [38]. The poles of the retarded Green's function, $\omega^{ \pm}$, give the spectrum of excitations, while the signs of their imaginary parts determine whether the proposed mean field steady state is stable (a positive imaginary part, $\Im\left(\omega^{ \pm}\right)>$ 0 , implies that the proposed steady state is unstable). Solving $\operatorname{det}\left(\left[D^{-1}\right]^{R}\right)=0$ for complex $\omega$ is equivalent to calculating the eigenvalues in linear response analysis $[33,35]$.

To calculate physical observables such as the luminescence and absorption spectra of the polariton system, we invert the Keldysh rotation to find the forwards $(<)$ and backwards $(>)$ Green's functions $[4,38,40]$ :

$$
\begin{aligned}
D^{<,>} & =\frac{1}{2}\left(D^{K} \mp\left(D^{R}-D^{A}\right)\right) ; \\
D^{<} & =-i\left\langle\psi_{f} \psi_{b}^{\dagger}\right\rangle .
\end{aligned}
$$

In energy $(\omega)$-momentum $(\mathbf{k})$ space, these give the incoherent luminescence

$$
L(\omega, \mathbf{k})=\frac{i}{2 \pi} D^{<}(\omega, \mathbf{k}),
$$

and absorption

$$
A(\omega, \mathbf{k})=\frac{i}{2 \pi} D^{>}(\omega, \mathbf{k})
$$

spectra in the steady state [4]. Note that since $D^{R, A, K}$ come from inverting Eq. (17) using Eqs. (18)-(20), they all contain $\operatorname{det}\left(\left[D^{-1}\right]^{R}\right)$ in the denominator; if this is zero while the numerators of $D^{<}$remain nonzero, then the luminescence diverges, indicating an instability of the chosen mean field solution towards a transition to a new phase.

In experiments polaritons are observed through the photon losses from the microcavities. The photon luminescence is obtained by extracting the photon part through multiplication by the (momentum dependent) photon fraction $C^{2}(\mathbf{k})$, defined in Eq. (2):

$$
L_{\mathrm{phot}}=C^{2}(\mathbf{k}) L_{l p}
$$

The spectral weight is defined as the difference between the absorption and luminescence. In terms of the Green's functions [54],

$$
S W(\omega, \mathbf{k})=A(\omega, \mathbf{k})-L(\omega, \mathbf{k})=\frac{i}{2 \pi}\left(D^{R}-D^{A}\right),
$$

or $1 /(2 \pi)$ times the spectral response of Ref. [41].

It should further be noted that recent work on the incoherently pumped polariton system, that considers the phase fluctuations to all orders with renormalization group analysis, has revealed that the long-wavelength, long-time behavior depends strongly on the anisotropy [55]. In particular, the isotropic limit of an infinite system has been shown to require an exact treatment of phase fluctuations, but a certain degree of spatial anisotropy leads the system to fall into the usual Gaussian fixed point, such as that considered in this paper. Our coherently pumped polariton system is intrinsically strongly anisotropic with the polariton dispersion substantially different along the two spatial directions perpendicular and parallel to the pump wave vector. This suggests that the Gaussian description will be sufficient for studying parametrically pumped polaritons in certain regimes and is certainly sufficient for the coherently pumped polaritons, where the Goldstone mode and so large phase fluctuations are absent. 


\section{E. Dimensionless units}

Throughout this paper, a flat exciton dispersion $\omega_{x}(\mathbf{k})=\omega_{x}$ is used. We work in a system of nondimensional units where the energies are rescaled such that $\Omega_{R} / 2=1$, and all the momenta or wave vectors (with $\hbar=k_{B}=1$ ) are rescaled to $\mathbf{k} \rightarrow \mathbf{k} / \sqrt{\Omega_{R} m_{c}}$. The key control parameters are the pump wave vector, which for OPO is typically in the range of $[51,56]$ $1.0 \mu \mathrm{m}^{-1}<\left|\mathbf{k}_{p}\right|<2.0 \mu \mathrm{m}^{-1}$, and the detuning of the pump energy away from the polariton dispersion $\omega_{p}-\omega_{l p}\left(\mathbf{k}_{p}\right)$. In particular for all results presented in this paper, we use $\mathbf{k}_{p}=(1.5,0)$ in dimensionless units along the $x$ direction, which corresponds to $\left|\mathbf{k}_{p}\right| \approx 1.677 \mu \mathrm{m}^{-1}$ for realistic $\Omega_{R}=$ $5 \mathrm{meV}$ and $m_{c}=0.25 \times 10^{-5} m_{e}$ [2,34]. The pump is chosen to be resonant with the lower polariton dispersion at the pump momentum, $\omega_{p}-\omega_{l p}\left(\mathbf{k}_{p}\right)=0$. This means that we avoid bistability in the pump mode by working in the so called 'optical limiter' regime [33,34,57]. With $\omega_{x}=0$, for $\mathbf{k}_{p}=(1.5,0), \omega_{p}=-0.380$. The polariton decay $\kappa_{l p}$ is chosen to be 0.05 in dimensionless units which corresponds to $\kappa_{l p}=0.125 \mathrm{meV}$. The exciton-exciton interaction strength $g_{\mathrm{x}}$ is implicitly rescaled to 1 which in turn applies a scaling to the fields.

\section{TRANSITION TO OPO}

As in previous theoretical studies of the OPO transition [28,32-34] a single mode mean field solution is considered first, i.e., assuming that only the mode with frequency and wave vector equal to that of the external pump is occupied, and we then investigate the appearance of the additional signal $\left(\mathbf{k}_{s}<\mathbf{k}_{p}, \omega_{s}<\omega_{p}\right)$ and idler $\left(\mathbf{k}_{i}>\mathbf{k}_{p}, \omega_{i}>\omega_{p}\right)$ modes with momenta and energies determined by momentum and energy conservation. The method described in the previous section is applied to this 'normal state' of coherently pumped polaritons.

\section{A. Mean field}

To get the simplest single-mode mean field solution, we make a plane wave ansatz for the solution at the pump frequency and wave vector in Eq. (14), restrict the interactions to remain within the pump mode, and calculate the steady state. The plane wave for the pump mode mean field in the rotating frame is just a constant, complex, amplitude:

$$
\psi_{p}^{\mathrm{mf}}=P \text {. }
$$

In the steady state, $\partial_{t} P=0$, this gives [32,34]:

$$
0=\left(\omega_{l p}\left(\mathbf{k}_{p}\right)-\omega_{p}-i \kappa_{l p}+V_{p p p}|P|^{2}\right) P+f .
$$

Note that from Eq. (26) onwards, instead of writing the momenta in the interaction [Eq. (6)], the mode (here $p$ ) index and the fluctuation signature ${ }^{ \pm}$are used.

Taking the modulus square of Eq. (26), the mode occupation $n_{p}=|P|^{2}$ is related to the pump strength $I_{p}=|f|^{2}$ as follows:

$$
I_{p}=\left(\omega_{l p}\left(\mathbf{k}_{p}\right)-\omega_{p}+V_{p p p} n_{p}\right)^{2} n_{p}+\kappa_{l p}^{2} n_{p} .
$$

Without any loss of generality, we can choose that the pumping field amplitude is real $\left(f=\sqrt{I_{p}}\right)$. A weak pump corresponds to a low $n_{p}$ while a strong pump has a high $n_{p}$. A typical behavior of $n_{p}$ as a function of pump power $I_{p}$ is shown in
Fig. 10 (the region where $n_{s}$ is zero corresponds to the pump only state discussed in this section).

\section{B. Green's functions and distribution matrix}

The inverse Green's functions are obtained by considering small fluctuations about the mean field. The pump mode with fluctuations can be written in the quantum-classical basis in the form of Eqs. (15) and (16) as follows:

$$
\begin{gathered}
\psi_{\mathbf{k}, c l}(\omega)=\sqrt{2} P \delta_{\mathbf{k}, \mathbf{0}} \delta_{\omega, 0}+\delta \psi_{\mathbf{k}, c l}(\omega), \\
\psi_{\mathbf{k}, q}=\delta \psi_{\mathbf{k}, q}(\omega),
\end{gathered}
$$

where the $\sqrt{2}$ in the classical part comes from the mean field solution being on the forwards branch of the closed time contour. As described in Sec. III D, only terms second order in the fluctuations are kept and there is an implicit summation over momenta. In the pump only state, the inverse retarded Green's functions in (17) are:

$$
\left[D^{-1}\right]^{R}(\omega, \mathbf{k})=\frac{1}{2}\left(\begin{array}{cc}
\omega-\alpha^{+}+i \kappa_{l p} & -V_{p_{+-}} P^{2} \\
-V_{p_{+-}} P^{* 2} & -\omega-\alpha^{-}-i \kappa_{l p}
\end{array}\right)
$$

where $\quad \alpha^{ \pm}=\omega_{l p}\left(\mathbf{k}_{p} \pm \mathbf{k}\right)-\omega_{p}+2 V_{p_{ \pm+}} n_{p}, \quad V_{p_{+-}}=$ $g_{\mathrm{x}} X^{2}\left(\mathbf{k}_{p}\right) X\left(\mathbf{k}_{p}+\mathbf{k}\right) X\left(\mathbf{k}_{p}-\mathbf{k}\right)$ and

$$
\left[D^{-1}\right]^{K}(\omega, \mathbf{k})=i \kappa_{l p}\left(\begin{array}{cc}
F_{\chi}\left(\omega+\omega_{p}\right) & 0 \\
0 & F_{\chi}\left(-\omega+\omega_{p}\right)
\end{array}\right) .
$$

Although the bath's distribution $F_{\chi}(\omega)$ can have any form, we choose $F_{\chi}(\epsilon)=2 n_{B}(\epsilon)+1$, where $n_{B}(\epsilon)$ is the Bose-Einstein distribution, to represent thermal modes outside of the cavity. As discussed in Sec. III B $F_{\chi}\left( \pm \omega+\omega_{p}\right) \approx 1$. Using Eqs. (18)(20) and (30)-(31),

$$
\begin{gathered}
D^{R}(\omega, \mathbf{k})=A\left(\begin{array}{cc}
-\omega-\alpha^{-}-i \kappa_{l p} & V_{p_{+-}} P^{2} \\
V_{p_{+-}} P^{* 2} & \omega-\alpha^{+}+i \kappa_{l p}
\end{array}\right), \\
D^{K}(\omega, \mathbf{k})=B\left(\begin{array}{cc}
v^{-} & \eta^{-} P^{2} \\
\eta^{+} P^{* 2} & v^{+}
\end{array}\right),
\end{gathered}
$$

where the following shorthand notation has been introduced

$$
\begin{aligned}
& v^{ \pm}=\left(\omega \mp \alpha^{ \pm}\right)^{2}+\kappa_{l p}^{2}+V_{p_{+-}}^{2} n_{p}^{2}, \\
& \eta^{ \pm}=\left(-\left(\alpha^{+}+\alpha^{-}\right) \pm 2 i \kappa_{l p}\right) V_{p_{+-}},
\end{aligned}
$$

and the prefactors are:

$$
\begin{aligned}
& A=\frac{1}{2 \operatorname{det}\left(\left[D^{-1}\right]^{R}(\omega, \mathbf{k})\right)}, \\
& B=\frac{-i \kappa_{l p}}{4\left|\operatorname{det}\left(\left[D^{-1}\right]^{R}(\omega, \mathbf{k})\right)\right|^{2}} .
\end{aligned}
$$

With the simple matrix structure, we use Eq. (20) to find the distribution matrix:

$$
F_{S}(\omega, \mathbf{k})=\left(\begin{array}{cc}
1 & \frac{-2 V_{p_{+}} P^{2}}{2 \omega-\alpha^{+}+\alpha^{-}} \\
\frac{-2 V_{p_{+}-} P^{* 2}}{2 \omega-\alpha^{+}+\alpha^{-}} & -1
\end{array}\right) .
$$

When $\omega-\left(\alpha^{+}-\alpha^{-}\right) / 2=0$, the distribution matrix diverges. Although $F_{s}$ can in general be very different to the equilibrium Bose-Einstein distribution, by comparing the two, we can 


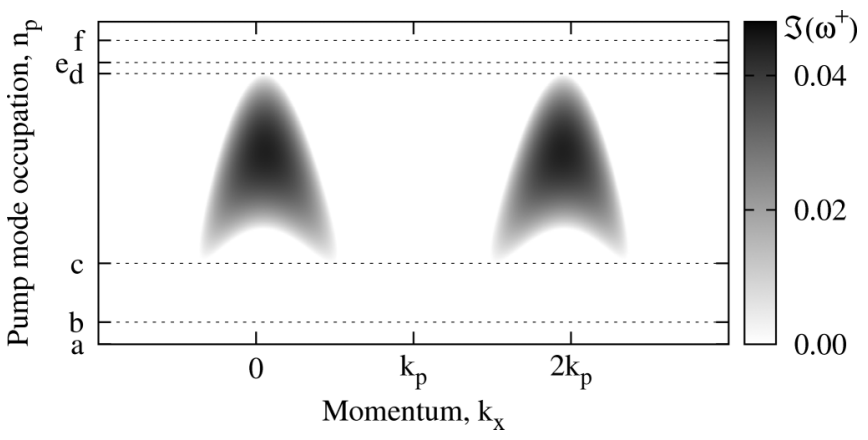

FIG. 2. Regions of unstable single-mode pump-only state, $\Im\left(\omega^{ \pm}\right)>0$, symmetric around the pump momentum $\mathbf{k}_{p}$. The letters and dotted lines correspond to the pump mode occupations used in Fig. 3 ( $\mathrm{a}$ is $n_{p}=1 \times 10^{-4}$ and cannot be resolved from the horizontal axis).

introduce an effective chemical potential as the energy at which the distribution function diverges:

$$
\mu_{\mathrm{eff}}(\mathbf{k})=\frac{\alpha^{+}-\alpha^{-}}{2} .
$$

\section{Zeros of $\left[D^{-1}\right]^{R}$ - spectra and chemical potential}

The inverse retarded Green's function $\left[D^{-1}\right]^{R}$ is related to the Bogoliubov matrix $L$ obtained by considering small fluctuations about the steady state [28,33,34]. In particular, $L(\mathbf{k})=$ $-2 \sigma_{z}\left(\left[D^{-1}\right]^{R}(0,-\mathbf{k})\right)$, and solving $\operatorname{det}\left(\left[D^{-1}\right]^{R}\left(\omega^{ \pm}, \mathbf{k}\right)\right)=0$ for $\omega^{ \pm}(\mathbf{k}) \in \mathbb{C}$ is equivalent to finding the eigenvalues of $L[4,34]$ :

$$
\omega^{ \pm}(\mathbf{k})=\frac{\alpha^{+}-\alpha^{-}}{2}-i \kappa_{l p} \pm \frac{1}{2} \sqrt{\left(\alpha^{+}+\alpha^{-}\right)^{2}-4 V_{p_{+-}}^{2} n_{p}^{2}} .
$$

The real parts of $\omega^{ \pm}(\mathbf{k})\left[\Re\left(\omega^{ \pm}(\mathbf{k})\right)\right]$ correspond to the spectra of excitations while the signs of the imaginary parts of $\omega^{ \pm}(\mathbf{k})$ determine whether the proposed mean field solution is stable (if $\Im\left(\omega^{ \pm}(\mathbf{k})\right)>0$ at any momentum, the proposed single-mode solution is unstable).

The unstable region depends on the external drive which is related to the pump mode occupation by Eq. (27). After transforming back to the laboratory frame, Fig. 2 shows that there are two regions around $k_{x}=0$ and $k_{x}=2\left|\mathbf{k}_{p}\right|$ where the single mode becomes unstable to small fluctuations as the external drive strength is increased (decreased) above (below) some lower (upper) threshold value. For the parameters chosen, the stability diagram presented in Fig. 2 shows the instability towards an OPO state. It also demonstrates that there is no bistability in the pump occupation since all instabilities occur away from the pump momentum.

It is also instructive to examine the real and imaginary parts of $\operatorname{det}\left(\left[D^{-1}\right]^{R}(\omega, \mathbf{k})\right)$. Considering $\Re\left[\operatorname{det}\left(\left[D^{-1}\right]^{R}\left(\xi^{ \pm}, \mathbf{k}\right)\right)\right]=0$ gives:

$$
\xi^{ \pm}(\mathbf{k})=\frac{\alpha^{+}-\alpha^{-}}{2} \pm \frac{1}{2} \sqrt{\left(\alpha^{+}+\alpha^{-}\right)^{2}+4\left(\kappa_{l p}^{2}-V_{p_{+-}}^{2} n_{p}^{2}\right)},
$$

while the requirement for the imaginary part of the determinant of the retarded inverse Green's function being zero corresponds to the same condition as the divergence of the distribution $F_{s}$ discussed in the previous section, i.e., $\Im\left[\operatorname{det}\left(\left[D^{-1}\right]^{R}\left(\mu_{\mathrm{eff}}, \mathbf{k}\right)\right)\right]=0$.

In Fig. 3, we plot $\Re\left(\omega^{ \pm}(\mathbf{k})\right), \Im\left(\omega^{ \pm}(\mathbf{k})\right), \xi^{ \pm}(\mathbf{k})$ and $\mu_{\text {eff }}(\mathbf{k})$ for a range of stable pump mode occupations. First the case of a weak pump (low $n_{p}$ ) is considered and the instability threshold is approached from below, as shown in the top row of Fig. 3. The imaginary parts of the complex eigenvalues $\Im\left(\omega^{ \pm}(\mathbf{k})\right)$ start to split and the real parts combine in four distinct regions, leading to the double tails when the pump state first becomes unstable, as seen in Fig. 2. When the four maxima in the imaginary parts of the complex eigenvalues first appear, two are located near to the pump momentum, one at a much higher and one at a much lower momentum. As the transition is approached, their values grow and those that were below the pump momentum move towards each other and the $k_{x}=0$ point, while those that were above the pump momentum move towards $k_{x}=2\left|\mathbf{k}_{p}\right|$. Note that close to the lower threshold (c in Fig. 2) there are two distinct momenta $k_{x}$ for the signal and two for the idler states, where the instability develops.

For a strong pump (high $n_{p}$ ), the pump mode becomes stable to small fluctuations again, defining an 'upper threshold.' At the upper threshold the instability develops at an unique momentum for signal and idler states. The behaviors above the upper threshold are shown in the bottom row of Fig. 3. Just above the upper threshold, there are only two places where $\Im\left(\omega^{+}(\mathbf{k})\right) \neq \Im\left(\omega^{-}(\mathbf{k})\right)$ : one near $k_{x}=0$ and one near $k_{x}=$ $2\left|\mathbf{k}_{p}\right|$. As the pump strength is further increased these peaks eventually disappear while the real parts of the eigenvalues $\left[\Re\left(\omega^{ \pm}(\mathbf{k})\right)\right]$ separate and become increasingly close to the poles $\left[\xi^{ \pm}(\mathbf{k})\right]$.

In general, $\xi^{ \pm}(\mathbf{k})$ (solid blue lines in Fig. 3) pinch together at the momenta where $\Im\left(\omega^{ \pm}(\mathbf{k})\right)$ is closest to 0 . The values of $\xi^{ \pm}(\mathbf{k})$ are very close to $\Re\left(\omega^{ \pm}(\mathbf{k})\right)$ apart from where $\Im\left(\omega^{ \pm}(\mathbf{k})\right)$ split [or differ from $\Im\left(\omega^{ \pm}(\mathbf{k})\right)=-\kappa$ ]. At these points, the effective chemical potential is equal to the real parts of the eigenvalues. The phase transition happens where the real and imaginary parts of the determinant of the inverse retarded Green's function become zero simultaneously, which indicates diverging luminescence in the normal state. This happens precisely when $\mu_{\mathrm{eff}}(\mathbf{k})=\xi^{ \pm}(\mathbf{k})$. In this sense the OPO phase transition happens in an analogous way to an equilibrium BEC phase transition: the effective chemical potential $\mu_{\text {eff }}(\mathbf{k})$ (red dashed line in Fig. 3) moves closer to the energies of the system defined by $\xi^{ \pm}(\mathbf{k})$ (solid blue lines in Fig. 3) as the density is increased, and the OPO transition takes place when $\mu_{\text {eff }}(\mathbf{k})$ reaches $\xi^{ \pm}(\mathbf{k})$.

To show clearly what happens across the instability threshold, we examine the behavior of $\Re\left(\omega^{ \pm}(\mathbf{k})\right), \Im\left(\omega^{ \pm}(\mathbf{k})\right), \xi^{ \pm}(\mathbf{k})$ and $\mu_{\text {eff }}(\mathbf{k})$ over a range of pump mode occupations for specific characteristic momenta. Since the transition to the OPO regime is of interest, we choose three characteristic momenta in the laboratory frame: $k_{x}=\left|\mathbf{k}_{p}\right|, 0$ and $\left|2 \mathbf{k}_{p}\right|$, i.e., the locations of the pump and expected signal and idler states. As can be seen in Fig. 4 , at the pump, $\Im\left(\omega^{ \pm}\right)<0$ and $\mu_{\text {eff }} \neq \xi^{ \pm}$at any $n_{p}$, i.e., there is no instability at the pump momentum at any occupation. This means that with the choice of tuning the pump to the lower polariton dispersion the system is in the optical limiter and not the bistable regime [32,33]. In contrast, for $k_{x}=0$ the effective chemical potential $\mu_{\text {eff }}$ decreases as the density is increased and crosses $\xi^{ \pm}$in two places indicating the 

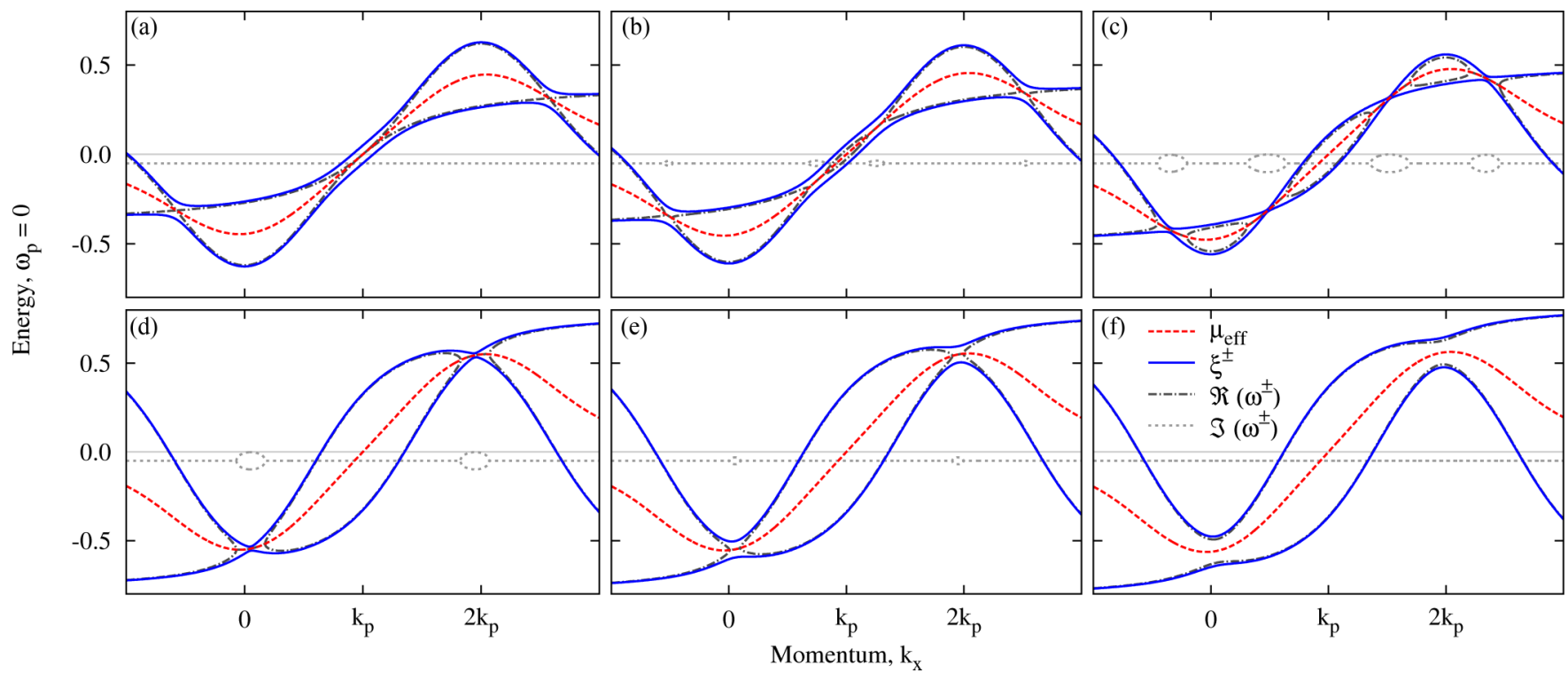

FIG. 3. Solutions to $\operatorname{det}\left(\left[D^{-1}\right]^{R}\right)=0$ for the stable pump mode occupations indicated in Fig. 2. Red dotted: $\mu_{\text {eff }}$ from $\Im\left(\operatorname{det}\left(\left[D^{-1}\right]^{R}\left(\mu_{\text {eff }}, \mathbf{k}\right)\right)\right)=0$ where $\mu_{\text {eff }} \in \mathbb{R}$; solid blue: $\xi^{ \pm}$from $\Re\left(\operatorname{det}\left(\left[D^{-1}\right]^{R}\left(\xi^{ \pm}, \mathbf{k}\right)\right)\right)=0$ where $\xi^{ \pm} \in \mathbb{R} ;$ dark gray dashed: $\Re\left(\omega^{ \pm}\right)$and gray dashed: $\Im\left(\omega^{ \pm}\right)$from $\operatorname{det}\left(\left[D^{-1}\right]^{R}\left(\omega^{ \pm}, \mathbf{k}\right)\right)=0$ where $\omega^{ \pm} \in \mathbb{C}$. Top row: approaching lower threshold from below: a) $n_{p}=1 \times 10^{-4}$; b) $n_{p}=0.02$; c) $n_{p}=0.073$. Bottom row: increasing $n_{p}$ above 'upper threshold': d) $n_{p}=0.245$; e) $n_{p}=0.255$; f) $n_{p}=0.275$.

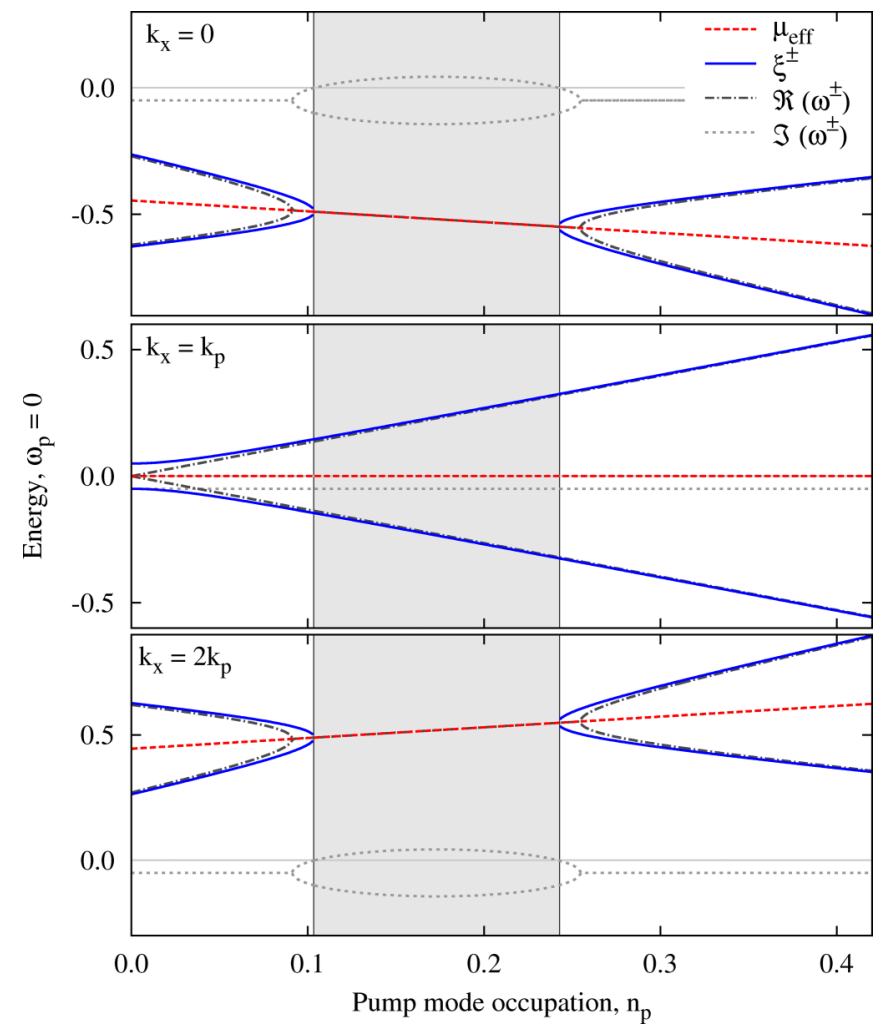

FIG. 4. As Fig. 3 but at selected momenta. The unstable region is indicated in gray. Top: $k_{x}=0$, i.e., at the expected signal, the chemical potential $\mu_{\text {eff }}$ decreases with increasing $n_{p}$ to cross $\xi^{ \pm}$when $\Im\left(\omega^{+}\right)=0$; middle: $k_{x}=\left|\mathbf{k}_{p}\right|, \mu_{\text {eff }}$ never crosses $\xi^{ \pm}$and $\Im\left(\omega^{ \pm}\right)<0$ for any $n_{p}$, i.e., there is no instability directly at the pump; bottom: $k_{x}=\left|2 \mathbf{k}_{p}\right|$, i.e., at the expected idler, the chemical potential $\mu_{\text {eff }}$ increases with increasing $n_{p}$ to cross $\xi^{ \pm}$when $\Im\left(\omega^{+}\right)=0$. upper and the lower threshold. Around the idler, $k_{x}=2\left|\mathbf{k}_{p}\right|$, the effective chemical potential is increasing with increasing density. It is also seen that the mode crossing occurs at the transition from a stable to an unstable region $\Im\left(\omega^{ \pm}\right)=0$. This behaviour is analogous to other bosonic condensations, where the phase transition is associated with the chemical potential crossing one of the energy modes [4,36]. It is interesting to note that the increasing chemical potential, and therefore the closest analogy, is around the expected idler.

This behavior of pinching and crossing can be seen clearly from the expressions for $\mu_{\mathrm{eff}}(\mathbf{k})$ [Eq. (33)], $\omega^{ \pm}(\mathbf{k})$ [Eq. (34)], and $\xi^{ \pm}(\mathbf{k})$ [Eq. (35)]. The imaginary parts of $\omega^{ \pm}$differ from $-i \kappa_{l p}$ when the discriminant in Eq. (34) is negative; the real part giving the spectra is then the same as the chemical potential in Eq. (33). Meanwhile, the first term in Eq. (35) is also the chemical potential, so when the discriminant is small the poles will be close to the chemical potential. This happens when $\left(\alpha^{+}+\alpha^{-}\right)-4 V_{p+-} n_{p} \approx-4 \kappa_{l p}^{2}$, which is exactly the condition for the imaginary part of one of the eigenvalues to become zero.

\section{Eigenvalues of the distribution matrix and effective temperature}

The concept of an effective temperature has been introduced in several driven-dissipative systems. Examples include glassy systems or shaken sand where there is a separation of time scales of the motion, and the effective temperature is related to the slow dynamics [58-61]. More recently, the extended fluctuation-dissipation relations that appear in the Keldysh formalism have been used to introduce an effective temperature for quantum driven-dissipative systems studied using Keldysh Green's functions $[41,62,63]$. In equilibrium, the bosonic 


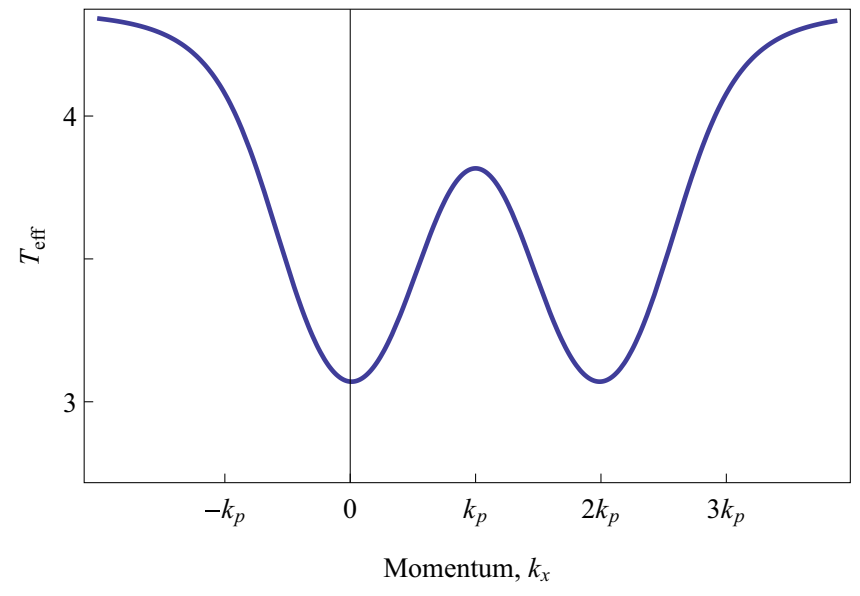

FIG. 5. The effective temperature, $T_{\text {eff }}(\mathbf{k})$ as defined by Eq. (37), with a local maximum at the applied pump and global minima at $k_{x}=$ $0,\left|2 \mathbf{k}_{p}\right|$. The pump mode occupation provides a purely multiplicative factor.

distribution matrix, $F_{s}$, is given by $\operatorname{coth}\left(\frac{\omega-\mu}{2 T}\right)\left(k_{B}=1\right)$, where $\mu$ is the chemical potential, i.e., $F_{s}$ diverges as $2 T / \omega$ when $\omega \rightarrow \mu$. This relation allows us to identify a low frequency effective temperature, $T_{\text {eff }}$, in this nonequilibrium system [41]. By examining the positive eigenvalue of $F_{s}\left(\lambda_{F_{s}}^{+}\right)$, an effective temperature can be defined as:

$$
\lambda_{F_{s}}^{+}=\frac{2 T_{\mathrm{eff}}}{\omega-\mu_{\mathrm{eff}}} .
$$

The eigenvalues of $F_{S}(\omega, \mathbf{k})[$ Eq. (32)] are:

$$
\lambda_{F_{s}}^{ \pm}(\omega, \mathbf{k})= \pm \sqrt{1+\frac{4 V_{p_{+-}}^{2} n_{p}^{2}}{\left(2 \omega-\alpha^{+}+\alpha^{-}\right)^{2}}}
$$

and the positive eigenvalue $\lambda_{F_{s}}^{+}(\omega, \mathbf{k})$ is considered. For $\omega \sim\left(\alpha^{+}-\alpha^{-}\right) / 2$, the second term dominates and

$$
\lambda_{F_{s}}^{+}(\omega, \mathbf{k}) \approx \frac{V_{p_{+-}} n_{p}}{\omega-\frac{\alpha^{+}-\alpha^{-}}{2}} .
$$

From Eq. (33), $\left(\alpha^{+}-\alpha^{-}\right) / 2$ is the effective chemical potential, $\mu_{\text {eff }}$ and, using Eq. (36), the low energy effective temperature

$$
T_{\mathrm{eff}}(\mathbf{k})=\frac{V_{p_{+-}} n_{p}}{2},
$$

which is plotted in Fig. 5 is obtained. The shape of $T_{\text {eff }}(\mathbf{k})$ is set by the $X^{4}$ contribution in $V_{p_{+-}}$and has minima at momenta $k_{x}=0$ and $k_{x}=\left|2 \mathbf{k}_{p}\right|$ in the laboratory frame. We note that in the OPO transition the "condensation" happens into signal and idler momenta close to the lowest effective temperature.

\section{E. Incoherent luminescence, absorption, and spectral weight}

When the single mode ansatz is stable, $D^{R}, D^{A}$, and $D^{K}$ are found using Eqs. (18)-(20) and the incoherent luminescence, absorption and spectral weight around the pump-mode state are

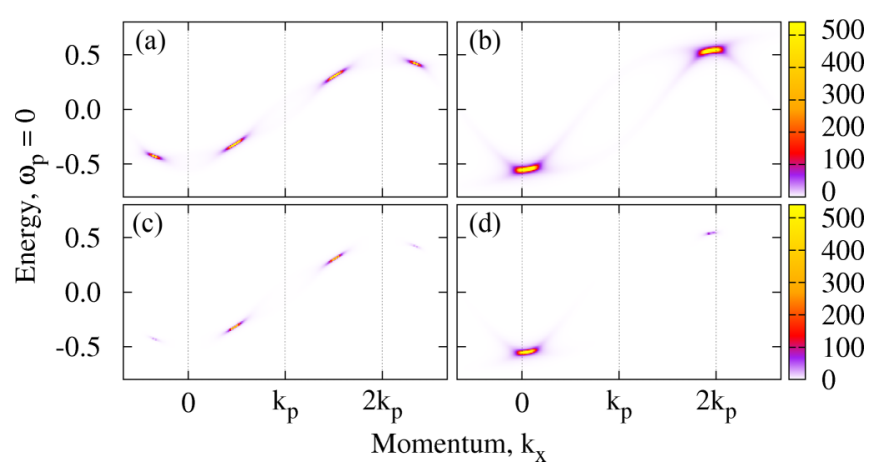

FIG. 6. Incoherent polariton (top) and photon (bottom) luminescence near the instability thresholds. Left: below lower threshold $n_{p}=0.073$; right: above upper threshold $n_{p}=0.245$.

calculated using Eqs. (21)-(23) and (25). For the remainder of this section, we consider the pump mode occupations of Figs. 3(c) and 3(d), close to the border of the unstable region, and all momenta are in the laboratory frame.

The incoherent luminescence shows peaks corresponding to the locations of the splitting in the imaginary parts of the eigenvalues $\Im\left(\omega^{ \pm}\right)$in Fig. 3 . When the pump is weak, below the lower threshold, four peaks appear in around the pump mode, as shown in Fig. 6(a). Above the 'upper threshold,' Fig. 6(b), there are only two peaks centered near $k_{x}=0$ and $k_{x}=\left|2 \mathbf{k}_{p}\right|$. This is consistent with the behavior of the eigenvalues in Figs. 2 and 3 .

One effect of assuming the same polariton decay rate at all momenta is to make the intensity peaks in the polariton luminescence appear symmetric about the pump mode which reflects the pairwise scattering process. In experiments, only the photonic component of polaritons can be measured and so the signal, which is more photonlike, appears stronger than the idler $[26,56]$. Thus, in the lower panels of Fig. 6, we rescale the luminescence according to the photon fraction [Eq. (24)] and the photon luminescence is stronger at low momenta, as expected. The absorption follows the same general pattern as the luminescence, but is generally stronger on the upper branch of the spectrum.

It is also interesting to examine the spectral weight [Eq. (25)]. As seen in Figs. 7(c) and 7(d), there are regions of negative spectral weight where the luminescence is greater than the absorption. Above the upper threshold, the spectral weight is negative below the chemical potential, as is usually the case. At weak pumping, the spectral weight is only very weakly negative, this occurs for energies below the chemical potential and away from the peaks in the luminescence. In Fig. 7(c), the range of the negative part of the spectral weight is greatly reduced for visibility.

Finally, we integrate the luminescence over energy and it is plotted as a function of two-dimensional momentum with $\mathbf{k}=\left(k_{x}, k_{y}\right)$ in Fig. 9. What looked as four peaks in the $(\omega$, $k_{x}$ ) plots (Figs. 6 and 7) was a signature of a ring structure for the signal and idler. At low pump powers close to the lower threshold the pump-only state becomes unstable to a 


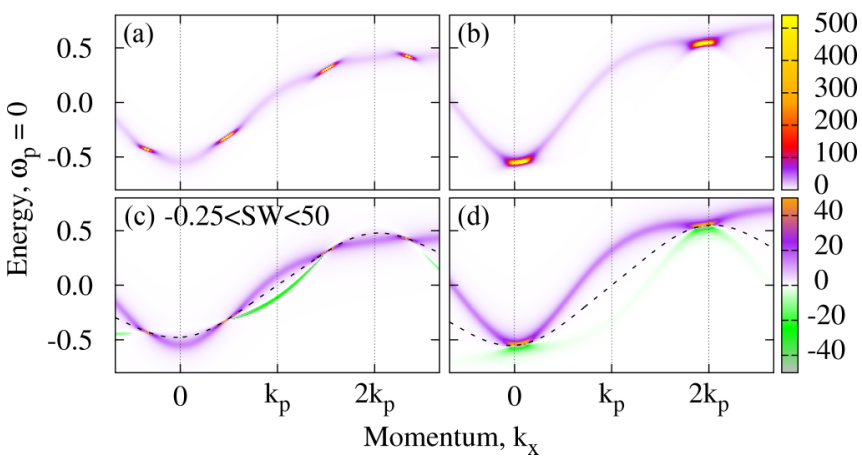

FIG. 7. Polariton absorption (upper) and spectral weight (lower) near the instability thresholds. Left: below lower threshold $n_{p}=$ 0.073; right: above upper threshold $n_{p}=0.245$. The scales for positive spectral weight are the same, at weak pumping, there is only weak negative spectral weight and the range is curtailed for visibility. The dashed line in the lower panels is the effective chemical potential $\mu_{\text {eff }}(\mathbf{k})$ of Fig. 3.

signal state with a ring shape in momentum. For large pump occupations, just above the 'upper threshold,' the instability develops at a unique momentum $\mathbf{k}_{s}=\left(k_{s}, 0\right)$ and so only two distinct peaks associated with developing signal and idler states are observed. However, there is no distinct signal momentum when the pump mode first becomes unstable, but there is instead initial growth at a range of momenta on a ring $\left|\mathbf{k}_{s}-\left(\Delta k_{x}, 0\right)\right|=k_{s}-\Delta k_{x}$ before a single momentum value dominates. The OPO transition can therefore be described using a distinct pair of new modes if the pump is decreased through the 'upper threshold' of the instability.

In Fig. 8, the incoherent luminescence is plotted for all momenta $\mathbf{k}=\left(k_{x}, 0\right)$ and energies below the pump energy (around the signal only). For weak pumping, the two peaks are broad and the one at the lower energy is weaker than the one at higher energy. Comparing this to Fig. 6(a), this shows that the peak at higher momentum dominates, which is consistent with the weaker luminescence on the side of the ring away from the pump in Fig. 9. Above the 'upper threshold,' the peak

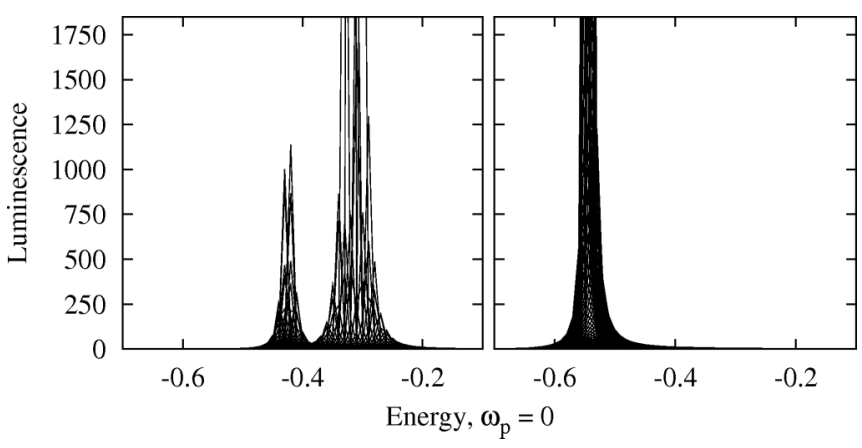

FIG. 8. Incoherent luminescence at energies below $\omega_{p}$ for a large rang of momenta. Left: $n_{p}=0.073$, below the lower threshold, the peak at higher energy dominates; right: $n_{p}=0.245$ above the upper threshold, the single peak is at a lower energy and is much narrower than the two peaks present for a weaker pump.

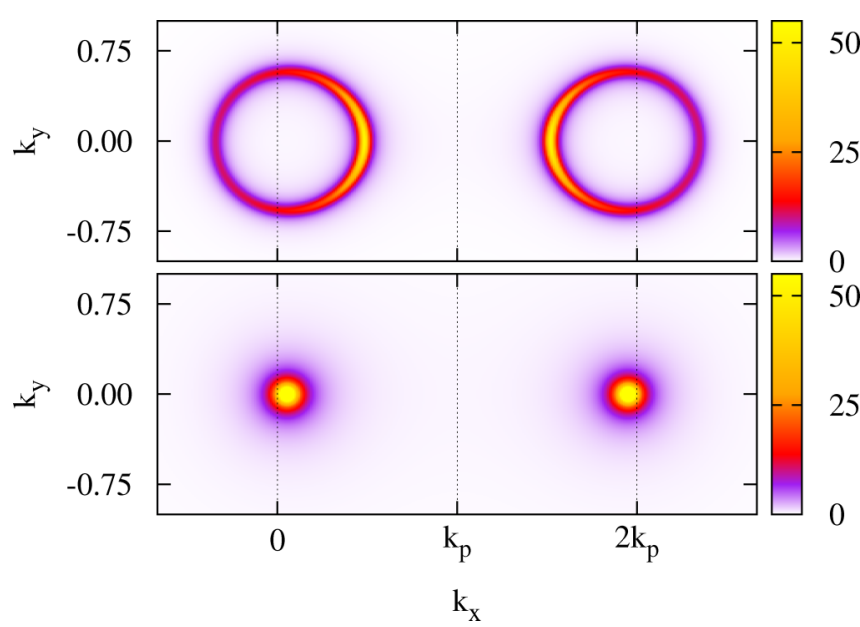

FIG. 9. Incoherent polariton luminescence integrated over energy and plotted in 2-D momentum space. Top: $n_{p}=0.073$; bottom: $n_{p}=$ 0.245 .

in the luminescence is narrower in energy signaling a phase transition to a single pair of signal-idler modes.

\section{ABOVE OPO THRESHOLD}

In the previous section the precursor of the OPO transition which manifested itself by the appearance of large occupations near $\mathbf{k}=0$ and $\mathbf{k}=2 \mathbf{k}_{p}$ was examined. Above the upper threshold, it had a particularly simple structure where the unstable regions gave a single signal mode, while at the lower threshold the signal develops on a ring in 2-D momentum space. Here we extend the analysis to the regime where the pump-only state mean field solution discussed before is unstable to small fluctuations. The next level of complexity is considered by including two additional modes in the mean field ansatz.

\section{A. OPO states and action}

To examine the behavior for parameters where the pumponly mean field solution is unstable, we divide the field into three subspaces in energy and momenta around the signal, pump and idler states such that $\psi=\psi_{s}+\psi_{p}+\psi_{i}$. The general idea is to include two additional modes, the signal $s$ and idler $i$, into the mean field ansatz, where each mode is restricted to momenta $\mathbf{q}_{j}+\mathbf{k}_{p}=\mathbf{k}_{s}, \mathbf{k}_{p}, \mathbf{k}_{i}$ [32,34,35], and then look at fluctuations around this solution. Due to the complicated nature of the instabilities when the pump strength is increased towards the lower threshold (low $n_{p}$ ), this simple mean field ansatz is valid in the region of stronger pump occupations closer to the upper threshold, where the OPO transition is expected to occur in a straightforward manner, i.e., where the peaks in the luminescence are located at two distinct momenta only with $k_{y}=0$, as shown in the lower panel of Fig. 9.

The full Keldysh action, Eq. (8), after integrating out the polariton decay bath, where the fields have been formally divided into three subspaces around signal, pump, and 
idler, is:

$$
\begin{aligned}
S_{O P O}= & \int d t\left(-\sqrt{2} f\left(\bar{\psi}_{p, q}+\psi_{p, q}\right)+\sum_{j=s, p, i}\left[\bar{\psi}_{j, c l}\left(i \partial_{t}-\omega_{l p}\left(\mathbf{k}_{j}+\mathbf{k}_{p}\right)+\omega_{p}-i \kappa_{l p}\right) \psi_{j, q}+\bar{\psi}_{j, q}\left(i \partial_{t}-\omega_{l p}\left(\mathbf{k}_{j}+\mathbf{k}_{p}\right)\right.\right.\right. \\
& \left.\left.+\omega_{p}+i \kappa_{l p}\right) \psi_{j, c l}\right]-\left\{\sum_{j=s, p, i} \frac{V_{j j j j}}{2} \bar{\psi}_{j, c l} \bar{\psi}_{j, q}\left(\psi_{j, c l}^{2}+\psi_{j, q}^{2}\right)+V_{s i s i}\left[\left(\bar{\psi}_{s, c l} \bar{\psi}_{i, c l}+\bar{\psi}_{s, q} \bar{\psi}_{i, q}\right)\left(\psi_{s, c l} \psi_{i, q}+\psi_{s, q} \psi_{i, c l}\right)\right]\right. \\
& +\sum_{j=s, i} V_{p j p j}\left[\left(\bar{\psi}_{p, c l} \bar{\psi}_{j, c l}+\bar{\psi}_{p, q} \bar{\psi}_{j, q}\right)\left(\psi_{p, c l} \psi_{j, q}+\psi_{p, q} \psi_{j, c l}\right)\right]+\frac{V_{p p s i}}{2}\left[2\left(\bar{\psi}_{s, c l} \bar{\psi}_{i, c l}+\bar{\psi}_{s, q} \bar{\psi}_{i, q}\right) \psi_{p, c l} \psi_{p, q}\right. \\
& \left.\left.\left.+\left(\bar{\psi}_{s, c l} \bar{\psi}_{i, q}+\bar{\psi}_{s, q} \bar{\psi}_{i, c l}\right)\left(\psi_{p, c l}^{2}+\psi_{p, q}^{2}\right)\right]+ \text { H.c. }\right\}\right)+\sum_{j=s, p, i} 2 i \kappa_{l p} \iint d t d t^{\prime} \bar{\psi}_{j, q}(t) F_{\chi}\left(t-t^{\prime}\right) \psi_{j, q}\left(t^{\prime}\right)
\end{aligned}
$$

where the fields for each mode $m$ are implicitly of the form $\psi_{m,\{c l, q\}}(t)$. The interaction coefficients now have four indices that indicate exactly which modes are involved in each scattering process.

\section{B. Mean field}

Taking the functional derivatives with respect to all $\psi$ fields in Eq. (38) and setting them to zero leads to the set of mean field equations analogous to Eq. (14). A mean field ansatz formed of three plane waves is chosen $[32,34,35]$. Written relative to the pump as before, the nonzero classical saddle-point fields are:

$$
\begin{aligned}
\psi_{s, c l}^{\mathrm{sp}}(t, \mathbf{x}) & =\sqrt{2} S e^{i \tilde{\omega} t} e^{-i \tilde{\mathbf{k}} \cdot \mathbf{x}}, \\
\psi_{p, c l}^{\mathrm{sp}}(t, \mathbf{x}) & =\sqrt{2} P, \\
\psi_{i, c l}^{\mathrm{sp}}(t, \mathbf{x}) & =\sqrt{2} I e^{-i \tilde{\omega} t} e^{i \tilde{\mathbf{k}} \cdot \mathbf{x}},
\end{aligned}
$$

with the signal and idler energies $\omega_{s, i}=\omega_{p} \mp \tilde{\omega}$ and momenta $\mathbf{k}_{s, i}=\mathbf{k}_{p} \mp \tilde{\mathbf{k}}$. The mode amplitudes $S, P, I$ are the mean field amplitudes and so have the prefactor $\sqrt{2}$ as discussed in Sec. III C. The general saddle-point form of any mode in the quantum-classical basis is:

$$
\psi_{m, c l}^{\mathrm{sp}}(t, \mathbf{x})=\sqrt{2} M e^{-i \omega_{m} t} e^{i \mathbf{q}_{m} \cdot \mathbf{x}} ; \quad \psi_{m, q}^{\mathrm{sp}}=0
$$

where, given the gauge transformation to the pump frame, the momenta are: $\mathbf{q}_{s}=-\tilde{\mathbf{k}}, \mathbf{q}_{p}=\mathbf{0}, \mathbf{q}_{i}=\tilde{\mathbf{k}}$ and energies: $\omega_{s}=-\tilde{\omega}, \omega_{p}=0$ and $\omega_{i}=\tilde{\omega}$ in what follows.

We could also substitute the new mean field ansatz, where the three modes of interest defined in Eq. (39) are included explicitly,

$$
\psi^{\mathrm{mf}}(t, \mathbf{x})=S e^{i \tilde{\omega} t} e^{-i \tilde{\mathbf{k}} \cdot \mathbf{x}}+P+I e^{-i \tilde{\omega} t} e^{i \tilde{\mathbf{k}} \cdot \mathbf{x}}
$$

directly into the general cGPE, Eq. (14), and take the steady state with $\partial_{t} P=\partial_{t} S=\partial_{t} I=0$. This gives three complex equations that can be solved to give the signal energy $\omega_{s}=-\tilde{\omega}$ and the complex mode amplitudes $S, P, I$ [32]. Some of the interaction terms introduce modes outside of the three mode ansatz; these are discarded. The cGPEs for each of the modes, after substitution of the three mode ansatz are [32]

$$
\begin{gathered}
\Xi_{s} S+V_{s p p i} P^{2} I^{*}=0, \\
\Xi_{p} P+2 V_{s p p i} S P^{*} I+f=0, \\
\Xi_{i} I+V_{s p p i} S^{*} P^{2}=0,
\end{gathered}
$$

where the shorthand

$$
\begin{aligned}
\Xi_{m}= & \omega_{l p}\left(\mathbf{q}_{m}+\mathbf{k}_{p}\right)+2\left(V_{m m s s} n_{s}+V_{m m p p} n_{p}+V_{m m i i} n_{i}\right) \\
& -\omega_{m}-\omega_{p}-V_{m m m m} n_{m}-i \kappa_{m},
\end{aligned}
$$

and $|P|=n_{p},|S|=n_{s},|I|=n_{i}$ has been used. Since the polariton decay is constant, $\kappa_{s}=\kappa_{i}=\kappa_{p}=\kappa_{l p}$. The steady state requires that the signal and idler momenta are specified [34]; we use the simplest choice of $\mathbf{k}_{s}=\mathbf{0}$ so $\mathbf{k}_{i}=2 \mathbf{k}_{p}$, although in experiments $\mathbf{k}_{s}$ is usually small but finite [14,51]. In the OPO regime, the occupation of the pump mode is depleted due to scattering into the signal and idler modes. The complex mode amplitudes can be considered to have the form $M=|M| e^{i \theta_{m}}$. The phase of the pump mode is locked to the external pump and can be determined from Eq. (41), but there is a phase freedom in the choice of the signal and idler; their phase difference is chosen spontaneously at each realisation of an experiment $[35,64]$. In the calculation of the mean field, the phase of one of these modes can be chosen freely. We choose the signal to be real and the idler phase is then determined by the steady state equations [Eqs. (40)-(42)]. This phase freedom means that a shift of the signal phase, e.g., $\theta_{s} \rightarrow \theta_{s}+\Delta \theta$, would be accompanied by a simultaneous change in the idler phase in the opposite direction, $\theta_{i} \rightarrow \theta_{i}-\Delta \theta$, while the equations of motion remain unchanged [35]. This phase freedom leads to the appearance of a gapless Goldstone mode that is not present in the pump-only configuration.

\section{Inverse Green's functions}

The inverse Green's functions for the OPO state are calculated as in the pump only case $\left(\psi_{m, c l} \rightarrow \psi_{m, c l}^{s p}+\delta \psi_{c l}^{m}, \psi_{m, q} \rightarrow\right.$ $\left.\delta \psi_{q}^{m}\right)$, and give $6 \times 6$ matrices due to the presence of three modes. Using the form of the fluctuations defined in Eqs. (15) and (16), the mean field plus fluctuations in the Keldysh quantum-classical basis is:

$$
\begin{aligned}
\psi_{\mathbf{k}, c l}(\omega)= & \sqrt{2} S \delta_{\mathbf{k},-\tilde{\mathbf{k}}} \delta_{\omega,-\tilde{\omega}}+\delta \psi_{\mathbf{k}-\tilde{\mathbf{k}}, c l}^{s}(\omega-\tilde{\omega}) \\
& +\sqrt{2} P \delta_{\mathbf{k}, \mathbf{0}} \delta_{\omega, 0}+\delta \psi_{\mathbf{k}, c l}^{p}(\omega) \\
& +\sqrt{2} I \delta_{\mathbf{k}, \tilde{\mathbf{k}}} \delta_{\omega, \tilde{\omega}}+\delta \psi_{\mathbf{k}+\tilde{\mathbf{k}}, c l}^{i}(\omega+\tilde{\omega})
\end{aligned}
$$

and

$$
\psi_{\mathbf{k}, q}(\omega)=\delta \psi_{\mathbf{k}-\tilde{\mathbf{k}}, q}^{s}(\omega-\tilde{\omega})+\delta \psi_{\mathbf{k}, q}^{p}(\omega)+\delta \psi_{\mathbf{k}+\tilde{\mathbf{k}}, q}^{i}(\omega+\tilde{\omega}),
$$


where the energies and momenta of the modes appear as offsets to the fluctuations. The inverse Green's functions can be written compactly as [35]:

$$
\left[D^{-1}\right]^{R}(\omega, \mathbf{k})=\frac{1}{2}\left(\begin{array}{cc}
M(+) & Q(+) \\
Q^{*}(-) & M^{*}(-)
\end{array}\right)
$$

with the elements of the submatrices $M, Q$ :

$$
\begin{aligned}
M_{m, n}( \pm)= & \delta_{m, n}\left(\omega_{m} \pm \omega-\omega_{l p}\left(\mathbf{k}_{m}^{ \pm}\right)+\omega_{p}+i \kappa_{l p}\right) \\
& -2 \sum_{r, t=1}^{3} \delta_{m+r, n+t} V_{m^{ \pm}, n^{ \pm}, r, t} \psi_{r}^{\mathrm{mf} *} \psi_{t}^{\mathrm{mf}}, \\
Q_{m, n}( \pm)= & -\sum_{r, t=1}^{3} \delta_{m+n, r+t} V_{m^{ \pm}, n^{ \pm}, r, t} \psi_{r}^{\mathrm{mf}} \psi_{t}^{\mathrm{mf}},
\end{aligned}
$$

where $\quad m^{ \pm}=\mathbf{k}_{m}^{ \pm}=\mathbf{q}_{m}+\mathbf{k}_{p} \pm \mathbf{k}, \quad \psi_{m}^{\mathrm{mf}}=\psi_{m, c l} / \sqrt{2} \quad$ and $m, n, r, t \in\{1,2,3\} \rightarrow\{S, P, I\} .\left[D^{-1}\right]^{R}(\omega, \mathbf{k})$ is related to the linear response matrix as in the pump only case. The inverse Keldysh Green's function, $\left[D^{-1}\right]^{K}(\omega, \mathbf{k})$, is similar to the one for pump-only state [Eq. (31)] with diagonal elements:

$$
K_{m, n}( \pm)=i \kappa_{l p} F_{\chi}\left(\omega_{m}+\omega_{p} \pm \omega\right) \delta_{m, n},
$$

and

$$
\left[D^{-1}\right]^{K}(\omega, \mathbf{k})=\left(\begin{array}{cc}
K(+) & 0 \\
0 & K(-)
\end{array}\right) .
$$

Taking the determinant of Eq. (43), and solving $\operatorname{det}\left(\left[D^{-1}\right]^{R}(\omega, \mathbf{k})\right)=0$ for $\omega^{j} \in \mathbb{C}$ gives the modes of the system $\omega^{j}$. With the three signal, idler, and pump mean field modes there are now six poles. We consider an example of a stable OPO near the upper threshold as identified in Fig. $10\left(I_{p}=9.016 I_{t h}\right)$. The real and imaginary parts of the eigenvalues of Eq. (43) are plotted in Fig. 11 showing that the steady state is stable $(\Im(\omega)<0)$, and that the Goldstone mode, characterised by $\Im(\omega) \rightarrow 0$ and $\mathfrak{R}(\omega) \rightarrow 0$ for $k \rightarrow 0$,

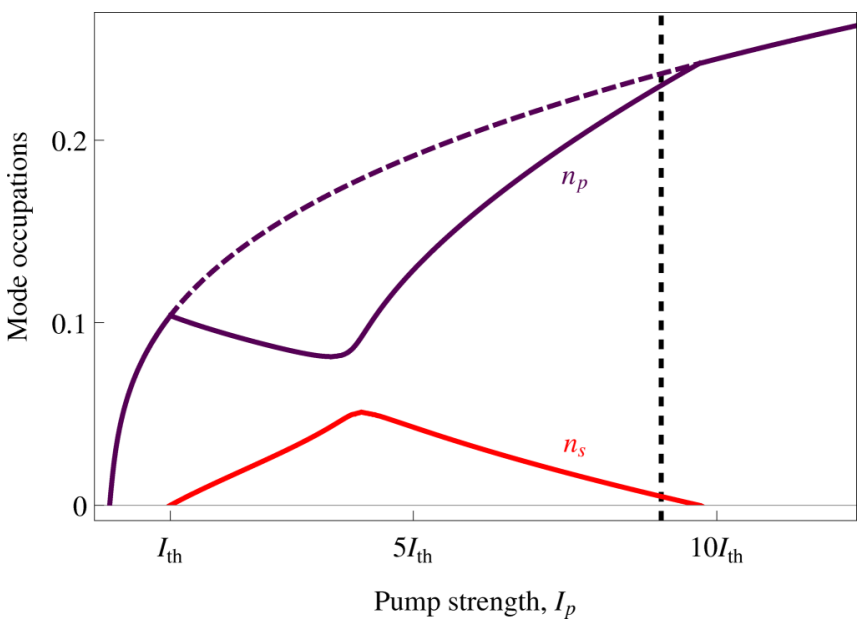

FIG. 10. Signal, $n_{s}$ (red), and pump, $n_{p}$ (purple, the dashed part is the pump only ansatz within the OPO region) mode occupations within the OPO regime for $\mathbf{k}_{p}=(1.5,0)$ and $\mathbf{k}_{s}=(0,0)$. The dashed vertical line is the pump strength considered for Figs. 11-14. The idler occupation $n_{i}$ is the same as the signal occupation for constant polariton decay $[29,30,32]$.

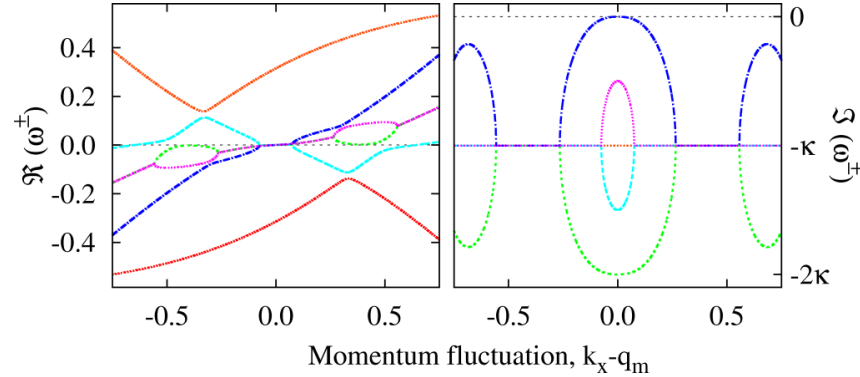

FIG. 11. Real $\Re\left(\omega^{j}\right)$ and imaginary parts $\Im\left(\omega^{j}\right)$ of the eigenvalues for the OPO state at pump strength: $I_{p}=9.016 I_{t h}$. The dark blue curve corresponds to the Goldstone mode. Since all the imaginary parts of the complex poles of the inverse retarded Green's function, $\omega^{ \pm}$are negative, the OPO ansatz is stable.

is present [35]. The Goldstone mode is associated with the spontaneous symmetry breaking of the phase freedom of the difference in signal and idler phases in the OPO regime. To remain within the three mode ansatz, it is assumed that the fluctuations in each mode are close in momenta and energy to that mode and the momentum range for plotting the results is therefore restricted to $k_{x}-q_{m}=\left(k_{p}-k_{s}\right) / 2$.

In Fig. 12, the very central region of the spectra is plotted. Although in Fig. 11 the real parts of the spectra appear flat in the limit $\omega \rightarrow 0, \mathbf{k} \rightarrow 0$, in Fig. 12, it is clear that although $\mathbf{k}_{s}=(0,0)$, the spectra are still sloped [35].

\section{Luminescence around the OPO states}

For the stable OPO state, the incoherent luminescence coming from fluctuations around the three mode ansatz is calculated using Eqs. (18)-(22). In Fig. 13, the contributions to the polariton luminescence around the signal, pump, and idler modes are considered separately and the spectra $(\Re(\omega)$ from linear response) overlaid. We can see variations in the occupations of the different branches according to the mode considered. For example, the outermost branches with increasing energy as the momentum of fluctuations increase are only noticeably occupied around the pump mode, while the parts of these branches characterised by decreasing energy with increasing momentum contribute to the luminescence around the signal mode for negative momentum of fluctuations and around the idler for positive momentum. The divergence

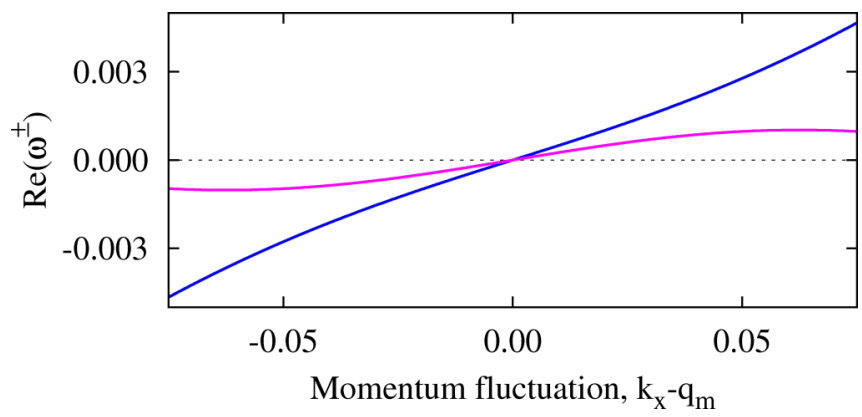

FIG. 12. The real parts of the spectra in a very small region around $\delta k=0$ showing that, although $\mathbf{k}_{s}=(0,0)$, there is still a finite slope of the Goldstone mode. 

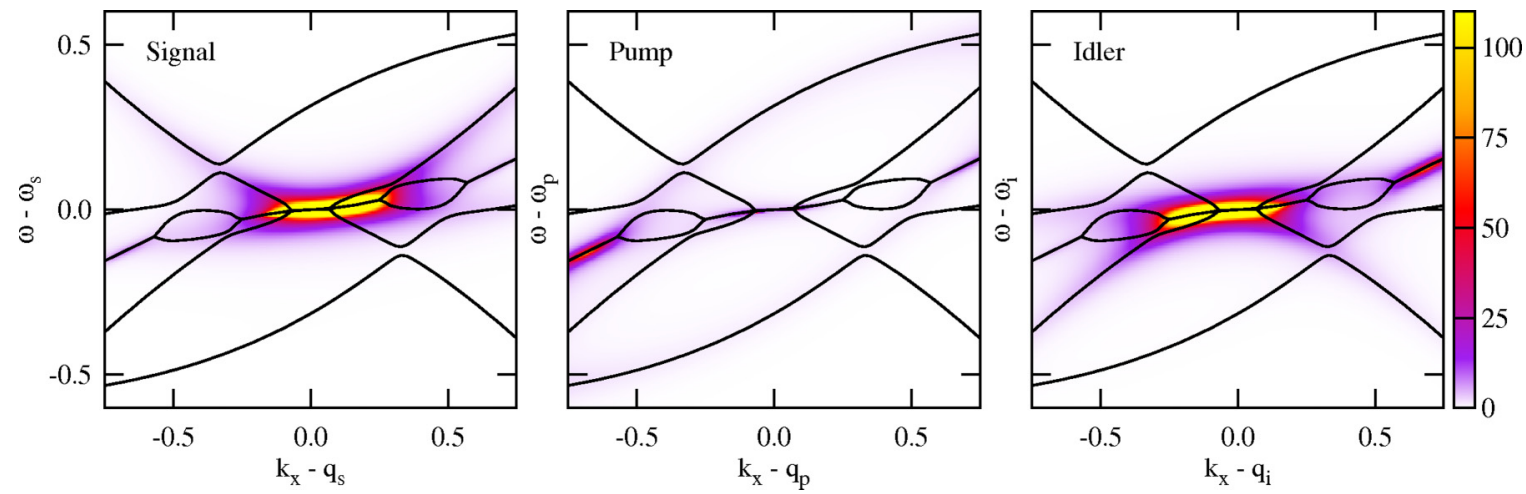

FIG. 13. Incoherent polariton luminescence about the three OPO states with the spectra $(\Re(\omega))$ overlaid. The Goldstone mode dominates the signal and idler states, where the luminescence is strong near the modes $\left(\omega_{s, i}, k_{s, i}\right)$, while the incoherent luminescence around the pump is much weaker.

caused by the Goldstone mode at $\omega_{s, i}, k_{x}=0$ leads to significant peaks close to the signal and idler states. There is only a weak peak in the incoherent luminescence around the pump mode $\left(\omega_{p}, k_{x}=0\right)$, which is due to the secondary splitting in the imaginary parts of the eigenvalues (central (blue/green) lines in the right hand side of Fig. 11): in this case, since the imaginary part is not zero, the luminescence does not diverge.

In the 'normal state', we saw that the peaks in the luminescence (Fig. 6) coincided with the maximum values of the imaginary parts of the eigenvalues (Fig. 3). In the 'condensed state' the strongest peaks in the incoherent luminescence about the OPO states are associated with the Goldstone mode while weaker peaks are the features of where other pairs of the six $\Im(\omega)$ split.

In Fig. 14 the energy and momentum ranges of Fig. 13 are shifted to the modes $\left(k_{x}=0 \rightarrow q_{m}\right.$ and $\left.\omega_{m}=0 \rightarrow \omega_{p}=0\right)$ to create a full picture of the incoherent luminescence around the OPO. The photon parts are included for completeness, and

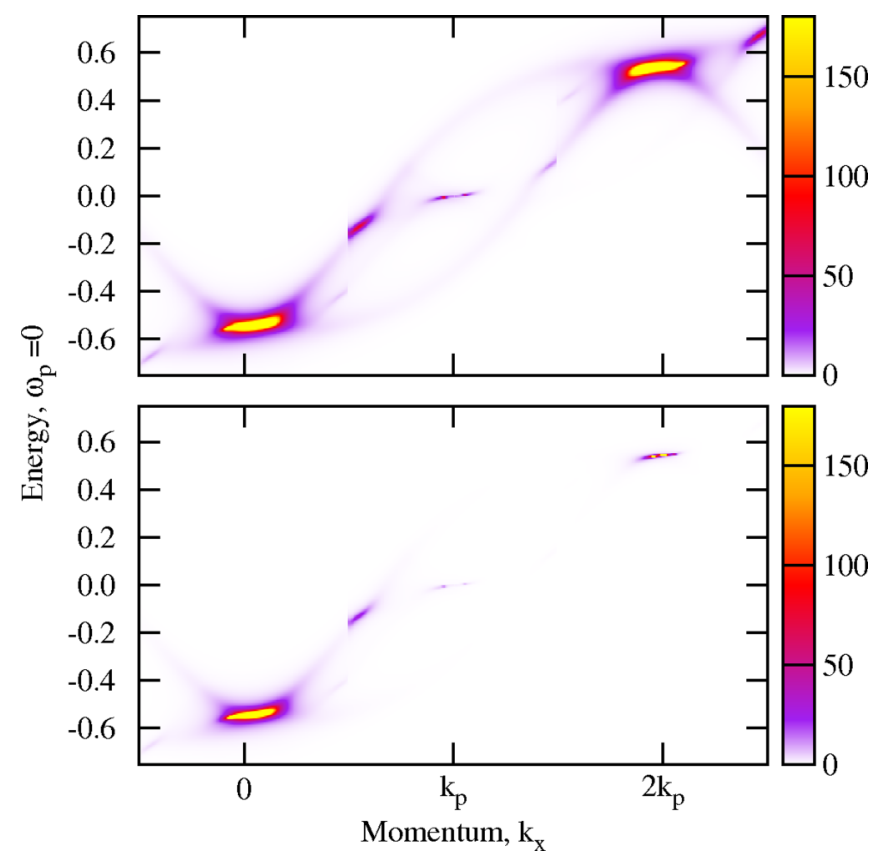

FIG. 14. The incoherent luminescence around the three OPO states combined. Top: polariton, Bottom: photon. to highlight the difference in visibility around the three modes due to the rate at which photons escape [56]. In particular, the weak peak at the pump mode becomes insignificant, and the incoherent luminescence is concentrated around the signal with a very small region around the idler mode, which are both due to the Goldstone mode.

\section{CONCLUSIONS}

In this paper, a Keldysh functional integral formulation for a coherently pumped polariton system coupled to a single incoherent decay bath has been developed and the polariton OPO transition studied. First we examined the 'normal state' of the pump only mode and calculated the spectra and instabilities in agreement with previous studies [32-34]. We have found that the system's nonequilibrium bosonic distribution function diverges at a momentum dependent energy which, by comparison with the equilibrium Bose-Einstein distribution, plays a role similar to the equilibrium chemical potential and which we call the effective chemical potential. In particular, considering how the 'normal state' becomes unstable, it was seen that at the instability the effective chemical potential crosses the energies of the normal modes at momenta associated with the signal and idler states. Moreover, at this effective chemical potential, the positive eigenvalue of the distribution matrix of the polariton system diverges as $1 / \omega$, which, by analogy to equilibrium, enabled us to identify an effective temperature for the low energy modes. The effective temperature is proportional to the polariton pump mode occupation, has global minima at $\mathbf{k}=\mathbf{0}$ and $\mathbf{k}=2 \mathbf{k}_{p}$, and a local maximum at $\mathbf{k}_{p}$. Remarkably, the system is 'condensing' into modes characterized by a temperature near the lowest possible.

To relate to experimentally measurable quantities, we calculated the incoherent luminescence, absorption, and spectral weight for two pump strengths, one just below the onset of instability and one where the 'normal state' becomes stable again. Close to the lower threshold the signal (idler) state develops on a momentum ring rather than at a single momentum as seen close to the upper threshold. The OPO regime can be described simply in terms of only three dominant momentum modes if the pump is decreased through the "upper threshold' of the instability. 
In the OPO regime, calculating the incoherent luminescence around the three modes shows that, although the spectra of small fluctuations are identical around each mode, the Goldstone mode affects only the signal and idler states and has little effect on the pump state. This is expected as the phase of the pump state is fixed by the driving process and therefore only small phase fluctuations are allowed. This is also in agreement with the observation that the vortex-anti-vortex pairs across the BKT transition are present in the signal and idler but not in the pump state [37]. It is clear that the occupations, as well as the forms of the excitation spectra determine the properties of a state.

\section{ACKNOWLEDGMENTS}

We thank F. M. Marchetti and A. Berceanu for helpful discussions. K.D. acknowledges help from J. M. Fellows with early versions of the Fortran code used. Support from EPSRC (Grant Nos. EP/I028900/2 and EP/K003623/2) is acknowledged.
[1] I. Carusotto and C. Ciuti, Rev. Mod. Phys. 85, 299 (2013).

[2] J. Keeling and N. G. Berloff, Contemp. Phys. 52, 131 (2011).

[3] M. H. Szymańska, J. Keeling, and P. B. Littlewood, Phys. Rev. Lett. 96, 230602 (2006).

[4] M. H. Szymańska, J. Keeling, and P. B. Littlewood, Phys. Rev. B 75, 195331 (2007).

[5] J. Keeling, M. H. Szymańska, and P. B. Littlewood, in Optical Generation and Control of Quantum Coherence in Semiconductor Nanostructures, Nanoscience and Technology, edited by G. Slavcheva and P. Roussignol (Springer, Berlin, Heidelberg, 2010), pp. 293-329.

[6] N. Proukakis, S. Gardiner, M. Davis, and M. Szymańska (eds.), Quantum Gases: Finite Temperature and Non-Equilibrium Dynamics (Imperial College Press, London, 2013).

[7] L. M. Sieberer, S. D. Huber, E. Altman, and S. Diehl, Phys. Rev. Lett. 110, 195301 (2013).

[8] M. Buchhold, P. Strack, S. Sachdev, and S. Diehl, Phys. Rev. A 87, 063622 (2013).

[9] J. Kasprzak, M. Richard, S. Kundermann, A. Baas, P. Jeambrun, J. M. J. Keeling, F. M. Marchetti, M. H. Szymańska, R. André, J. L. Staehli et al., Nature (London) 443, 409 (2006).

[10] R. Balili, V. Hartwell, D. W. Snoke, L. Pfeiffer, and K. West, Science 316, 1007 (2007).

[11] A. Amo, D. Sanvitto, F. P. Laussy, D. Ballarini, E. del Valle, M. D. Martin, A. Lemaître, J. Bloch, D. N. Krizhanovskii, M. S. Skolnick et al., Nature (London) 457, 291 (2009).

[12] A. Amo, J. Lefrère, S. Pigeon, C. Adrados, C. Ciuti, I. Carusotto, R. Houdré, E. Giacobino, and A. Bramati, Nat. Phys. 5, 805 (2009).

[13] D. Sanvitto, F. M. Marchetti, M. H. Szymańska, G. Tosi, M. Baudisch, F. P. Laussy, D. N. Krizhanovskii, M. S. Skolnick, L. Marrucci, A. Lemaître et al., Nat. Phys. 6, 527 (2010).

[14] A. C. Berceanu, L. Dominici, I. Carusotto, D. Ballarini, E. Cancellieri, G. Gigli, M. H. Szymańska, D. Sanvitto, and F. M. Marchetti, Phys. Rev. B 92, 035307 (2015).

[15] G. Nardin, G. Grosso, Y. Léger, B. Piętka, F. Morier-Genoud, and B. Deveaud-Plédran, Nat. Phys. 7, 635 (2011).

[16] E. Wertz, L. Ferrier, D. D. Solnyshkov, R. Johne, D. Sanvitto, A. Lemaître, I. Sagnes, R. Grousson, A. V. Kavokin, P. Senellart et al., Nat. Phys. 6, 860 (2010).

[17] D. Sanvitto, S. Pigeon, A. Amo, D. Ballarini, M. De Giorgi, I. Carusotto, R. Hivet, F. Pisanello, V. G. Sala, P. S. S. Guimaraes et al., Nat. Photon. 5, 610 (2011).

[18] G. Grosso, G. Nardin, F. Morier-Genoud, Y. Léger, and B. Deveaud-Plédran, Phys. Rev. Lett. 107, 245301 (2011).
[19] A. Amo, S. Pigeon, D. Sanvitto, V. G. Sala, R. Hivet, I. Carusotto, F. Pisanello, G. Leménager, R. Houdré, E. Giacobino et al., Science 332, 1167 (2011).

[20] J. O. Hamp, A. K. Balin, F. M. Marchetti, D. Sanvitto, and M. H. Szymańska, Europhys. Lett. 110, 57006 (2015).

[21] N. Bobrovska and M. Matuszewski, Phys. Rev. B 92, 035311 (2015).

[22] A. Chiocchetta and I. Carusotto, Europhys. Lett. 102, 67007 (2013).

[23] D. D. Solnyshkov, H. Tercas, K. Dini, and G. Malpuech, Phys. Rev. A 89, 033626 (2014).

[24] P. G. Savvidis, J. J. Baumberg, R. M. Stevenson, M. S. Skolnick, D. M. Whittaker, and J. S. Roberts, Phys. Rev. Lett. 84, 1547 (2000).

[25] R. M. Stevenson, V. N. Astratov, M. S. Skolnick, D. M. Whittaker, M. Emam-Ismail, A. I. Tartakovskii, P. G. Savvidis, J. J. Baumberg, and J. S. Roberts, Phys. Rev. Lett. 85, 3680 (2000).

[26] J. J. Baumberg, P. G. Savvidis, R. M. Stevenson, A. I. Tartakovskii, M. S. Skolnick, D. M. Whittaker, and J. S. Roberts, Phys. Rev. B 62, R16247 (2000).

[27] A. I. Tartakovskii, D. N. Krizhanovskii, D. A. Kurysh, V. D. Kulakovskii, M. S. Skolnick, and J. S. Roberts, Phys. Rev. B 65 , 081308 (2002).

[28] C. Ciuti, P. Schwendimann, and A. Quattropani, Phys. Rev. B 63, 041303 (2001).

[29] D. M. Whittaker, Phys. Rev. B 63, 193305 (2001).

[30] C. Ciuti, P. Schwendimann, and A. Quattropani, Semicond. Sci. Technol. 18, S279 (2003).

[31] I. Carusotto and C. Ciuti, Phys. Rev. Lett. 93, 166401 (2004).

[32] D. M. Whittaker, Phys. Rev. B 71, 115301 (2005).

[33] C. Ciuti and I. Carusotto, Phys. Status Solidi B 242, 2224 (2005)

[34] M. Wouters and I. Carusotto, Phys. Rev. B 75, 075332 (2007).

[35] M. Wouters and I. Carusotto, Phys. Rev. A 76, 043807 (2007).

[36] L. Pitaevskii and S. Stringari, Bose-Einstein Condensation, no. 116 in International Series of Monographs on Physics (Oxford University Press, New York, 2003).

[37] G. Dagvadorj, J. M. Fellows, S. Matyjaśkiewicz, F. M. Marchetti, I. Carusotto, and M. H. Szymańska, Phys. Rev. X 5, 041028 (2015).

[38] A. Kamenev, in Les Houches, Session LXXXI, 2004 Nanophysics: Coherence and Transport, edited by H. Bouchiat, Y. Gefen, G. Montambaux, and J. Dalibard (Elsevier, Amsterdam, 2005). 
[39] A. Altland and B. Simons, Condensed Matter Field Theory (Cambridge University Press, New York, 2010), 2nd ed.

[40] A. Kamenev, Field Theory of Non-Equilibrium Systems (Cambridge University Press, New York, 2011).

[41] E. G. D. Torre, S. Diehl, M. D. Lukin, S. Sachdev, and P. Strack, Phys. Rev. A 87, 023831 (2013).

[42] A.-W. de Leeuw, H. T. C. Stoof, and R. A. Duine, Phys. Rev. A 88, 033829 (2013).

[43] S. Gopalakrishnan, B. L. Lev, and P. M. Goldbart, Phys. Rev. A 82, 043612 (2010).

[44] J. Marino and S. Diehl, Phys. Rev. Lett. 116, 070407 (2016).

[45] M. Yamaguchi, K. Kamide, R. Nii, T. Ogawa, and Y. Yamamoto, Phys. Rev. Lett. 111, 026404 (2013).

[46] M. Yamaguchi, K. Kamide, T. Ogawa, and Y. Yamamoto, New J. Phys. 14, 065001 (2012).

[47] A. V. Kavokin, J. J. Baumberg, G. Malpuech, and F. P. Laussy, Microcavities (Oxford University Press, New York, 2007).

[48] J. J. Hopfield, Phys. Rev. 112, 1555 (1958).

[49] H. Deng, H. Haug, and Y. Yamamoto, Rev. Mod. Phys. 82, 1489 (2010).

[50] F. M. Marchetti and M. H. Szymańska, in Exciton Polaritons in Microcavities, Vol. 172 of Springer Series in Solid-State Sciences, edited by V. Timofeev and D. Sanvitto (Springer, Berlin, Heidelberg, 2012), pp. 173-213.

[51] R. Butté, M. S. Skolnick, D. M. Whittaker, D. Bajoni, and J. S. Roberts, Phys. Rev. B 68, 115325 (2003).
[52] L. D. Landau and E. M. Lifshitz, Quantum Mechanics (Pergamon Press, Oxford, 1965).

[53] I. Carusotto and C. Ciuti, Phys. Rev. B 72, 125335 (2005).

[54] F. M. Marchetti, J. Keeling, M. H. Szymańska, and P. B. Littlewood, Phys. Rev. B 76, 115326 (2007).

[55] E. Altman, L. M. Sieberer, L. Chen, S. Diehl, and J. Toner, Phys. Rev. X 5, 011017 (2015).

[56] W. Langbein, Phys. Rev. B 70, 205301 (2004).

[57] A. Baas, J. P. Karr, H. Eleuch, and E. Giacobino, Phys. Rev. A 69, 023809 (2004).

[58] C. Song, P. Wang, and H. A. Makse, Proc. Nat. Acad. Sci. U.S.A. 102, 2299 (2005).

[59] E. Bouchbinder and J. S. Langer, Phys. Rev. E 80, 031132 (2009).

[60] P. Sollich and S. Fielding, and P. Mayer, J. Phys.: Condens. Matter 14, 1683 (2002).

[61] L. F. Cugliandolo, J. Kurchan, and L. Peliti, Phys. Rev. E 55, 3898 (1997).

[62] L. M. Sieberer, S. D. Huber, E. Altman, and S. Diehl, Phys. Rev. B 89, 134310 (2014).

[63] M. F. Maghrebi and A. V. Gorshkov, Phys. Rev. B 93, 014307 (2016).

[64] A. Kavokin and G. Malpuech, in Cavity Polaritons, Vol. 32 of Thin Films and Nanostructures (Academic Press, San Diego, 2003), pp. 147-181. 